Please do not remove this page

RMIT

UNIVERSITY

\title{
A novel vehicle-based GNSS integrity augmentation system for autonomous airport surface operations
}

Suresh Bijjahalli, Suraj; Ramasamy, Subramanian; Sabatini, Roberto

https://researchrepository.rmit.edu.au/esploro/outputs/9921860616901341/filesAndLinks?institution=61RMIT_INST\&index=null

Suresh Bijjahalli, S., Ramasamy, S., \& Sabatini, R. (2017). A novel vehicle-based GNSS integrity augmentation system for autonomous airport surface operations. Journal of Intelligent and Robotic Systems: Theory and Applications, 87(2), 379-403. https://doi.org/10.1007/s10846-017-0479-8 Document Version: Accepted Manuscript

Published Version: https://doi.org/10.1007/s10846-017-0479-8 
Thank you for downloading this document from the RMIT Research Repository.

The RMIT Research Repository is an open access database showcasing the research outputs of RMIT University researchers.

RMIT Research Repository: http://researchbank.rmit.edu.au/

\section{Citation:}

Bijjahalli, S, Ramasamy, S and Sabatini, R 2017, 'A novel vehicle-based GNSS integrity augmentation system for autonomous airport surface operations', Journal of Intelligent and Robotic Systems: Theory and Applications, pp. 1-25.

See this record in the RMIT Research Repository at:

https://researchbank.rmit.edu.au/view/rmit:43596

Version: Accepted Manuscript

\section{Copyright Statement:}

(C) Springer Science+Business Media Dordrecht 2017

Link to Published Version:

https://dx.doi.org/10.1007/s10846-017-0479-8 


\title{
A NOVEL VEHICLE-BASED GNSS INTEGRITY AUGMENTATION SYSTEM FOR AUTONOMOUS AIRPORT SURFACE OPERATIONS
}

\author{
Suraj Bijjahalli• Subramanian Ramasamy• Roberto Sabatini*
}

\author{
School of Engineering - Aerospace Engineering and Aviation Discipline \\ Melbourne, VIC 3000, Australia
}

*roberto.sabatini@rmit.edu.au

\begin{abstract}
Autonomous vehicles equipped with integrity augmentation systems offer the potential to increase safety, efficiency and sustainability of airport ground operations. The model predictive behavior of these systems supports a timely detection of any deviations from the Required Navigation Performance (RNP), producing useful alerts for onboard mission management. Firstly, the system architecture of a Navigation and Guidance System (NGS) for autonomous airport surface vehicle operations based on Global Navigation Satellite System (GNSS) measurements is described. Subsequently, an integrity augmentation module is implemented in the NGS by modeling the key GNSS signal degradation phenomena including masking, multipath and signal attenuation. The GNSS integrity augmentation system is capable of monitoring the RNP and alerting the remote operator of the airport surface vehicle. The uniqueness of the presented system is that both caution and warning flags are produced based on prediction-avoidance and reaction-correction capabilities respectively. Additionally, the system is capable of issuing suitable steering commands to the onboard mission management system/remote ground base station operator in the event of GNSS signal degradations or losses. Multipath is modelled in detail using a ray tracing algorithm and the vehicle position error is computed as a function of relative geometry between the satellites, receiver antenna and reflectors
\end{abstract}

in realistic airport operation scenarios. Additionally, the surface vehicle dynamics and reflective surfaces of buildings are modelled in order to simulate a vehicle trajectory through a typical airport airside/aprons environment. Simulation case studies are performed to validate the mathematical models developed for the integrity augmentation system and the results corroborate the suitability of the proposed system to generate useful and timely integrity flags when GNSS is used as the primary means of navigation.

\section{Keywords:}

Global Navigation Satellite System · Avionics Based Augmentation System - Navigation and Guidance System · Airport Ground Operations · Autonomous Systems · Model Predictive Systems

\section{Introduction}

A steady growth in global and regional air traffic drives the demand for exploring new means of making airport operations safer, more efficient and more environmentally sustainable. A survey performed by the Flight Safety Foundation (FSF) estimates that 27,000 accidents cause 243,000 injuries globally every year. The accident and injury rates per 1000 departures calculated from this estimate are 1 and 9 respectively [1]. A lack of industry wide standards for ramp operations, coupled with the fact that safety on the ramp is handled primarily by 
This is the author pre-publication version. This paper does not include the changes arising from the revision, formatting and publishing processes. The final version that should be used for referencing is:

S. Bijjahalli, S. Ramasamy and R. Sabatini, "A Novel Vehicle-Based GNSS Integrity Augmentation System for Autonomous Airport Surface Operations", Journal of Intelligent \& Robotic Systems, pp. 1-25, 2017. DOI: 10.1007/s10846-017-0479-8

airlines, makes adherence to regulations inconsistent. Emerging solutions for aircraft tug operations including Taxibot $^{\mathrm{TM}}$ and SAFETug ${ }^{\mathrm{TM}}$ employ a tractor push for operating an aircraft in the airside of airports. Both manned and autonomous tugs are currently in use, with the autonomous tugs gaining significance owing to its features that support reduced human intervention. Robotic systems that perform ground operations autonomously or with minimal human intervention can facilitate uniform ramp policies and ensure adherence by eliminating factors such as human error, fatigue and boredom. Robotic vehicles have been previously implemented on a small scale at Heathrow airport as part of a Personal Rapid Transit (PRT) system [2].

Autonomous perception and control minimizes the need for an external infrastructure including guidance rails. The integration of autonomous vehicles with airside operations, however has not been extensively researched so far. Safety regulations enforced for airside operations constrain vehicle movement to precisely defined pathways making it necessary for robotic vehicles to demonstrate trusted autonomy (i.e., the vehicle must be able to determine whether data obtained from the on-board sensors is reliable or is corrupted by external error sources). A Navigation and Guidance System (NGS) using Carrier-Phase Differential Global Navigation Satellite System (CPD-GNSS) for localizing autonomous airport surface vehicles is presented. CPD-GNSS systems have previously been employed for localizing robotic systems with a high degree of precision. However, CPD-GNSS is generally susceptible to several failure modes that increase the Total System Error (TSE), leading to the requirement for 'system integrity'. In the context of GNSS receivers, integrity refers to the level of confidence that can be placed in the signal measurements and subsequent position solution. A well designed integrity augmentation system will be able to detect faults and alert the user in a timely manner to allow avoidance manoeuvres. A framework for an autonomous airport ground vehicle navigation system proposed in [3] highlights the necessity of GNSS integrity augmentation in order to meet the requirements of safe airside navigation. Integrity augmentation strategies that address GNSS signal degradations or losses have been implemented in the past, mainly targeting aircraft navigation and guidance. However, these techniques are not directly applicable to ground vehicle navigation [4-6]. Existing GNSS integrity augmentation methods include Ground-Based Augmentation Systems (GBAS), Satellite-Based Augmentation Systems (SBAS) and Aircraft-Based Augmentation Systems (ABAS). GBAS makes use of reference receivers located at precisely surveyed locations to compute integrity data ('Use/Do not use' messages) and pseudorange corrections, and disseminate them to nearby mobile receivers, typically via a Very High Frequency (VHF) link. GBAS leverages the fact that several GNSS pseudorange error components (satellite clock errors, ephemeris errors, atmospheric errors) are spatially correlated. SBAS is also a technique that depends on reference and mobile receivers sharing the same error in order to provide valid integrity alerts and corrections. SBAS exploits Geostationary Earth Orbit (GEO) satellites to expand the coverage area of integrity provision and pseudorange corrections. Both GBAS and SBAS incur a higher cost in terms of infrastructure and require a minimum number of visible satellites to function effectively. More importantly, these techniques cannot provide integrity in the presence of local GNSS errors like signal multipath, which dominates pseudorange error in complex ground environments [7]. ABAS monitors integrity using algorithms that process redundant GNSS signal measurements and onboard sensors. In several aviation applications, ABAS offers greater flexibility then GBAS/SBAS as it can be designed to address all error components of GNSS signals including local phenomena like multipath and antenna masking. This makes ABAS suitable for an extended range of integrity augmentation functionalities covering all operational flight phases (i.e., ground taxing, takeoff, en-route and landing). The system operates via separate principles from GNSS, and is therefore not subject to the same sources of error and interference. An Aircraft-Based Integrity Augmentation (ABIA) system is presented in [8-10], in which the GNSS signal degradations affecting manned/unmanned aircraft navigation is modelled to provide timely integrity alerts to pilots. This research broadens the spectrum of the ABIA concept, which is a type of ABAS, to ground transportation systems and the present paper specifically addresses the 
This is the author pre-publication version. This paper does not include the changes arising from the revision, formatting and publishing processes. The final version that should be used for referencing is:

S. Bijjahalli, S. Ramasamy and R. Sabatini, "A Novel Vehicle-Based GNSS Integrity Augmentation System for Autonomous Airport Surface Operations", Journal of Intelligent \& Robotic Systems, pp. 1-25, 2017. DOI: 10.1007/s10846-017-0479-8

implementation of model predictive features in airport surface vehicles. Causes of GNSS signal degradations in airport environments including masking, multipath and carrier-to-noise ratio are modelled and estimated. In particular, ray tracing methods have been adopted to model signal multipath $[11,12]$. Conventionally, ray tracing methods have been implemented for static applications where the receiver is assumed to be stationary. Therefore, there is an opportunity to explore the validity of this approach for assessing multipath in kinematic applications. Based on the above discussions, the key focus of this research is to design and implement an integrity augmentation system for ground vehicle GNSS receivers. The system is capable of detecting sources of signal degradation and initiating safety procedures autonomously. The approach can be applied to wheeled or tracked airport ground vehicles operating on the airside region that are designed for operations such as aircraft towing, catering, de-icing, cargo transport, debris detection and removal from runways. Integrity augmentation systems have been designed and implemented to provide integrity in the presence of GNSS faults (signal fading, antenna masking, multipath) occurring on aerial platforms. In ground vehicle navigation, these faults are attributed to sources that differ from those encountered in aerial navigation. GNSS faults in ground environments are analysed to design an integrity augmentation system that fits into a Communication, Navigation, and Surveillance (CNS) framework for airport surface operations as illustrated in Fig. 1. The NGS on board each airport ground vehicle delivers time-stamped position and velocity estimates to other ground vehicles, parked aircraft and to a central Ground Base System (GBS) via Lineof-Sight (LOS) wireless VHF datalink and Beyond LOS (BLOS) communication links. The wireless GBS comprises several modules that enhance the ground operator's situational awareness such as vehicle parameters and trajectory display, tactical map, video feed, communication console, etc. Visionbased sensors on the vehicle can enhance some of these functionalities. A vehicle command console with a Human-Machine Interface (HMI) is used to issue commands to the NGS. A generic four-wheeled vehicle dynamics model representative of an airside vehicle, such as a cargo truck is selected and presented in later sections along with an autonomous steering control law to validate the introduced mathematical models.

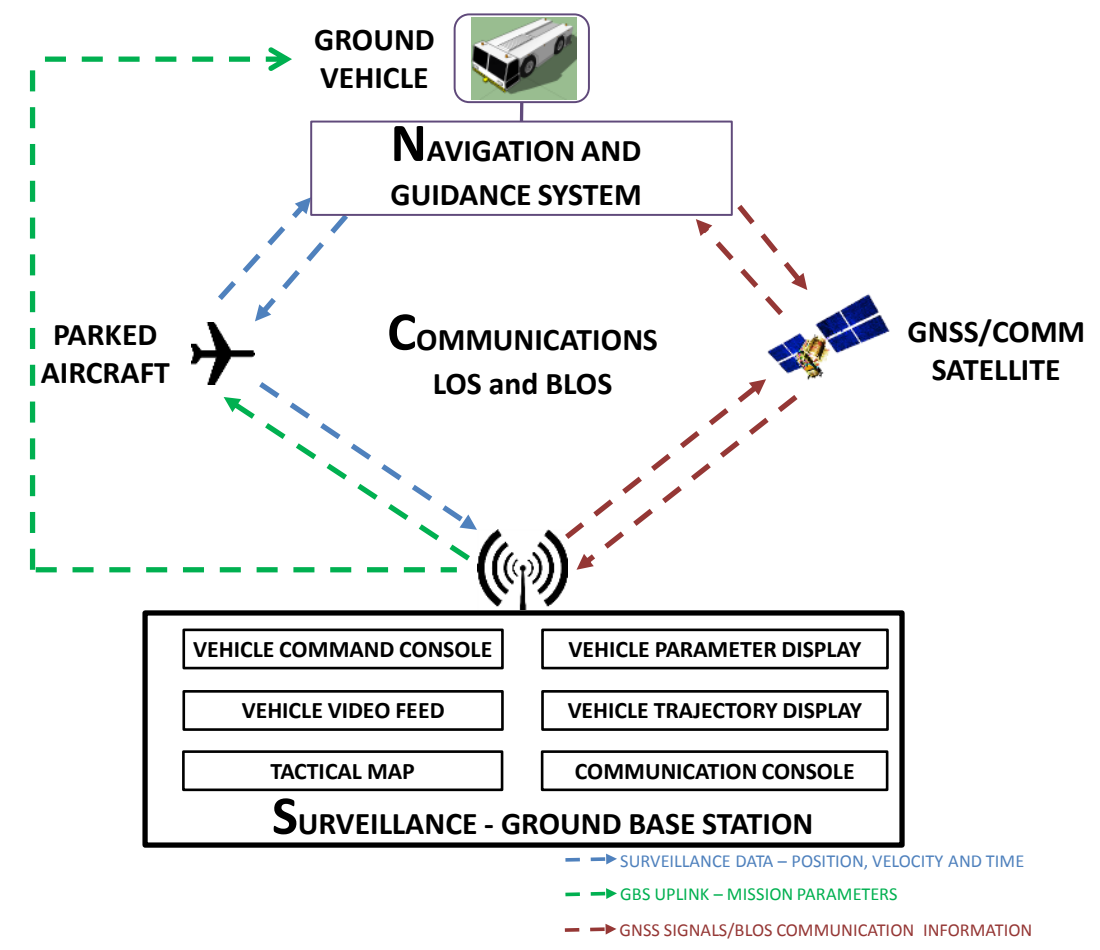

Fig. 1 CNS framework for autonomous airport surface operations 
This is the author pre-publication version. This paper does not include the changes arising from the revision, formatting and publishing processes. The final version that should be used for referencing is:

S. Bijjahalli, S. Ramasamy and R. Sabatini, "A Novel Vehicle-Based GNSS Integrity Augmentation System for Autonomous Airport Surface Operations", Journal of Intelligent \& Robotic Systems, pp. 1-25, 2017. DOI: 10.1007/s10846-017-0479-8

\section{Navigation and Guidance System}

Most autonomous systems are based on an architecture as described in $[13,14]$. The layers of these systems typically include [4,15-17] :

- A sensory layer including GNSS receivers, Inertial Navigation Systems (INS) and other navigation sensors.

- A perception layer comprising of a multisensor data fusion algorithm such as an Extended Kalman Filter (EKF) or Unscented Kalman Filter (UKF) to fuse GNSS and other sensory data to obtain an accurate best estimate of the vehicle's Position, Velocity and Attitude (PVA) measurements.

PVA estimation can be improved by augmenting GNSS information with differential measurements from a number of reference stations in order to compensate for atmospheric errors, and by measuring the phase of the incoming carrier wave signal. In theory, position estimation accurate to a few centimetres can be achieved by CPD-GNSS [18]. Additionally, CPD-GNSS can be leveraged on a multi antenna setup to obtain highly accurate attitude estimates, which can then be fused with INS attitude estimates [18,19], a concept that has been well researched for attitude determination, mainly in the aviation domain [20-23]. An NGS based on CPDGNSS is therefore a likely candidate to achieve the required positioning and attitude accuracy for autonomous ground navigation tasks. However, CPDGNSS based navigation is subject to error sources that can degrade the performance, and are dependent on:

- Satellite geometry, quantified by Dilution Of Precision (DOP);

- Atmospheric effects (ionospheric advance, tropospheric delay, attenuation and scintillation), which can either lower accuracy of performance;
- Antenna masking, which can potentially block signals;

- Multipath, which is dependent on the navigation environment.

DOP parameters are well defined as in $[18,19,24]$ and the receiver software is usually configured to ignore satellites that result in a poor DOP. Several models for quantifying atmospheric errors have been developed as described in $[18,19]$ to mitigate PVA degradations using suitable countermeasures. Antenna masking is directly related to the LOS between satellites and the receiver antennas, and this, in turn, is dependent on the airport environment and relative geometry between the antenna and various objects in the environment. Multipath, or signal reflections prior to arrival at the receiver can severely reduce PVA accuracy by inducing errors in the signal phase measurements, which are a function of the reflecting surface material, and the relative geometry between the receiver antenna and the reflector. An overall NGS architecture highlighting the various error sources, the sensor suite, a data-fusion module, an integrity augmentation system and a mission management system is illustrated in Fig. 2. The modelling of error sources associated with Global Positioning System (GPS), which is one of the most widely used GNSS constellations, enables us to understand their effect on position error, and allows for early detection of accuracy degradation, thereby boosting system integrity or trustworthiness. Corruption of the pseudorange measurements due to erroneous measurement of the Pseudo Random Noise (PRN) code or carrier-phase can lower the system accuracy to hazardous levels. Hence it is necessary to define accuracy thresholds or alert limits based on analytical modelling of GPS error sources. Since the positioning error cannot be deduced directly, the key challenge is to infer lowered accuracy for each satellite based on carrier-phase measurements, and accordingly issue alerts. 
This is the author pre-publication version. This paper does not include the changes arising from the revision, formatting and publishing processes. The final version that should be used for referencing is:

S. Bijjahalli, S. Ramasamy and R. Sabatini, "A Novel Vehicle-Based GNSS Integrity Augmentation System for Autonomous Airport Surface Operations", Journal of Intelligent \& Robotic Systems, pp. 1-25, 2017. DOI: 10.1007/s10846-017-0479-8

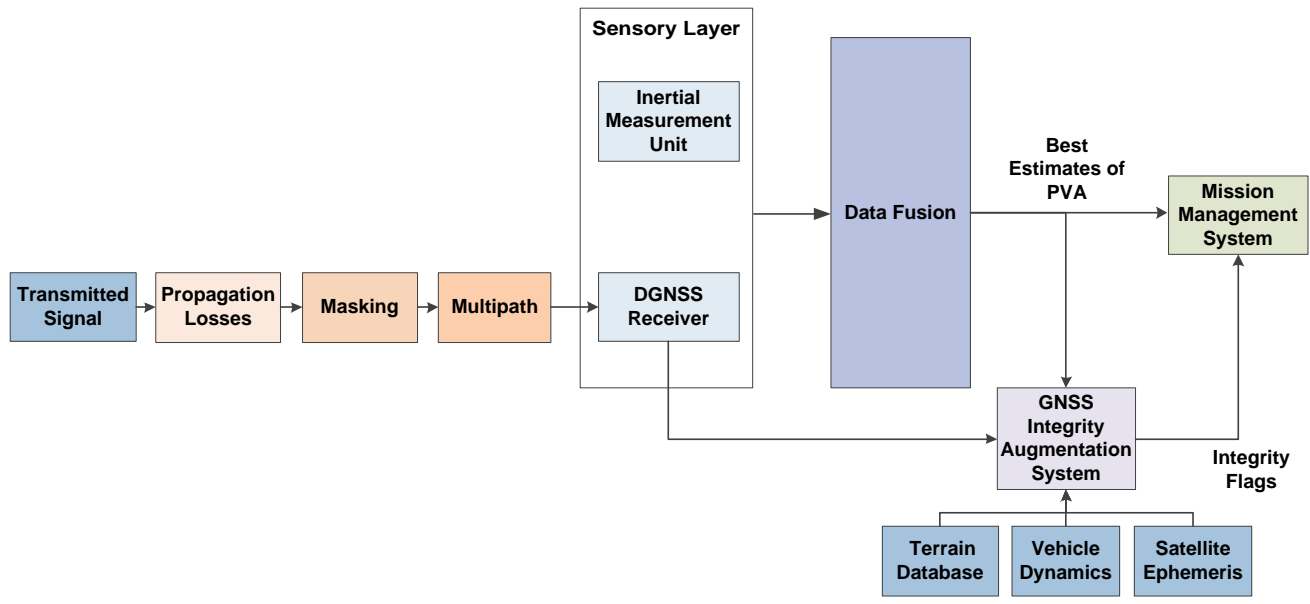

Fig. 2 System architecture

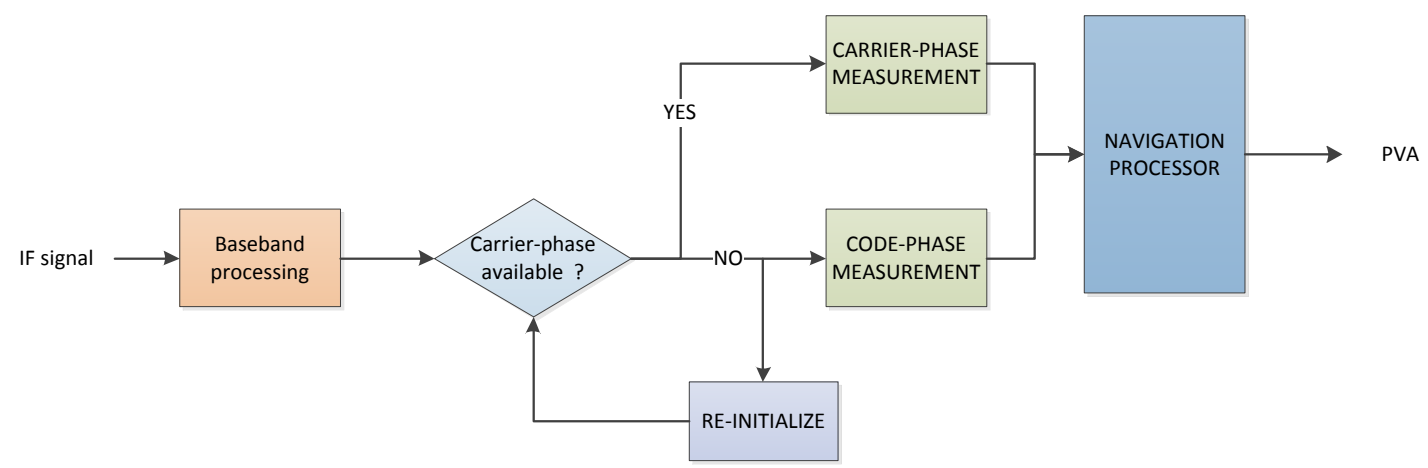

Fig. 3 Carrier/code phase measurement decision logic

The error sources described can potentially cause a loss-of-lock with satellite(s), resulting in a discontinuity in the receiver's carrier-phase measurement, a phenomenon known as 'cycle-slip'. A cycle-slip makes it necessary for the receiver to reinitialize of the algorithm used to measure carrierphase, a computationally intensive and timeconsuming process. The presence of buildings and other objects in the airport environment can increase the occurrence of cycle-slips. The proposed system therefore is capable of switching to code-range (C/A) measurements when carrier-phase measurements are affected by cycle-slips. In Fig. 3, the navigation processing module in the receiver uses carrier-phase measurements to compute PVA. If carrier-phase measurements are interrupted, the algorithm reinitializes the cycle-count for measuring carrierphase, while switching to a code-range measurement in parallel. Although code-range yields a lower accuracy, this capability to switch between measurements makes the system robust to loss-oflock with satellites.

\section{GNSS Integrity Augmentation}

In line with the above discussions, integrity is defined in [25] as the capability of the system to issue alerts when system accuracy degrades below a predefined 
This is the author pre-publication version. This paper does not include the changes arising from the revision, formatting and publishing processes. The final version that should be used for referencing is:

S. Bijjahalli, S. Ramasamy and R. Sabatini, "A Novel Vehicle-Based GNSS Integrity Augmentation System for Autonomous Airport Surface Operations", Journal of Intelligent \& Robotic Systems, pp. 1-25, 2017. DOI: 10.1007/s10846-017-0479-8

threshold. GNSS system integrity has been extensively studied in literature, the most notable is the Receiver Autonomous Integrity Monitoring (RAIM) [19], which computes the Horizontal and Vertical Protection Level (HPL/VPL) or the minimum position error that can be detected, given the measurement noise and satellite geometry. Carrier-phase based RAIM (CRAIM) extends the concept of RAIM to GNSS receivers that use carrierphase measurements to compute vehicular position. The primary drawbacks in CRAIM arise due to multipath, cycle-slips and ambiguity resolution [26]. Several approaches to CRAIM that differ with regard to how integer ambiguity is resolved are found in the literature [27-29]. A particularly robust solution is found in [26], wherein an integrity algorithm that used Double-Differenced (DD) pseudorange, DD widelane (L1-L2) and the L1 carrier-phase in conjunction with an EKF is presented.

Alternative approaches to detecting cycle-slips can be incorporated in integrity augmentation systems as described in [24,30-33]. Apart from GBAS and SBAS, integrity can be supported by systems on board the vehicle itself, referred to as ABAS, when implemented on an aircraft. An example of ABAS that processes variables including aircraft dynamics, antenna location and electromagnetic interference was implemented by Sabatini et al. [8], [34] to raise timely visual and/or aural alerts to the pilot in the event of a threat to GNSS integrity. The system has been demonstrated to have synergies with other safety-critical applications such as Sense-and-Avoid (SAA) used in unmanned systems [35]. A similar integrity augmentation system does not exist for autonomous ground navigation that could detect potential sources of signal degradation and issue timely alerts (both cautions and warnings) to planning and control modules. This would enable autonomous ground vehicles to be predictive rather than reactive to GNSS data degradations. The traditional integrity augmentation systems described above are aimed at providing integrity for aerial platforms. On the other hand, the components of positioning error in GNSSbased ground vehicle navigation are dominated by signal multipath from reflective objects close to the receiver antenna. The design of a ground vehicle integrity augmentation system is accordingly driven by an analysis of the GNSS signal errors unique to ground vehicle navigation. As previously mentioned, the ground vehicle integrity augmentation system presented in this paper adapts an ABIA system [8,34] to address GNSS integrity for ground vehicle navigation in complex environments. ABIA was originally intended to deliver integrity for aerial GNSS navigation by modelling the following GNSS error degradations:

- Antenna masking: In aerial navigation, GNSS integrity is commonly threatened by lowered satellite visibility owing to obscuration or masking of the receiver antenna by the aircraft body during dynamic manoeuvres. ABIA addressed this error mode in [8] by triggering alerts to the pilot/autopilot at threshold values of orientation angles (yaw, pitch, roll) to prevent reduced satellite visibility. But, in ground vehicle navigation, satellite visibility is dictated primarily by the presence of obstacles (buildings, trees, and other reflective surfaces) rather than vehicle manoeuvres.

- Signal propagation losses and multipath-induced fading: In aerial navigation, the Signal-to-Noise Ratio (SNR) at the receiver front-end is a function of free-space loss, atmospheric attenuation and the transmitter and receiver antenna gains. Multipath is primarily attributed to signal reflection from aircraft fuselage and wings prior to arrival at the antenna. Ground vehicle multipath, in contrast, introduces greater randomness owing to the fact that signal reflection occurs at reflective surfaces close to the receiver antenna. Signal fading in these circumstances is dependent on the angle of incidence of the signal and the material properties of the reflector.

- Multipath phase-error: Ground vehicle GNSS navigation is subject to error in signal code and carrier-phase measurement owing to signal multipath from reflectors located near the receiver, which ultimately biases the positioning solution. Therefore, the ABIA system cannot be directly applied to provide integrity for ground vehicle navigation. The integrity augmentation 
This is the author pre-publication version. This paper does not include the changes arising from the revision, formatting and publishing processes. The final version that should be used for referencing is:

S. Bijjahalli, S. Ramasamy and R. Sabatini, "A Novel Vehicle-Based GNSS Integrity Augmentation System for Autonomous Airport Surface Operations", Journal of Intelligent \& Robotic Systems, pp. 1-25, 2017. DOI: 10.1007/s10846-017-0479-8

system presented in this paper extends the envelope of the ABIA system to ground vehicles through detailed modelling of ground multipath.

Two categories of integrity alerts are defined as in [8]: caution flags and warning flags. The caution flag provides a model-predictive behaviour of the system, supporting the avoidance of signal degradations/losses. The prediction-avoidance capability of the integrity augmentation system is illustrated in Fig. 4.

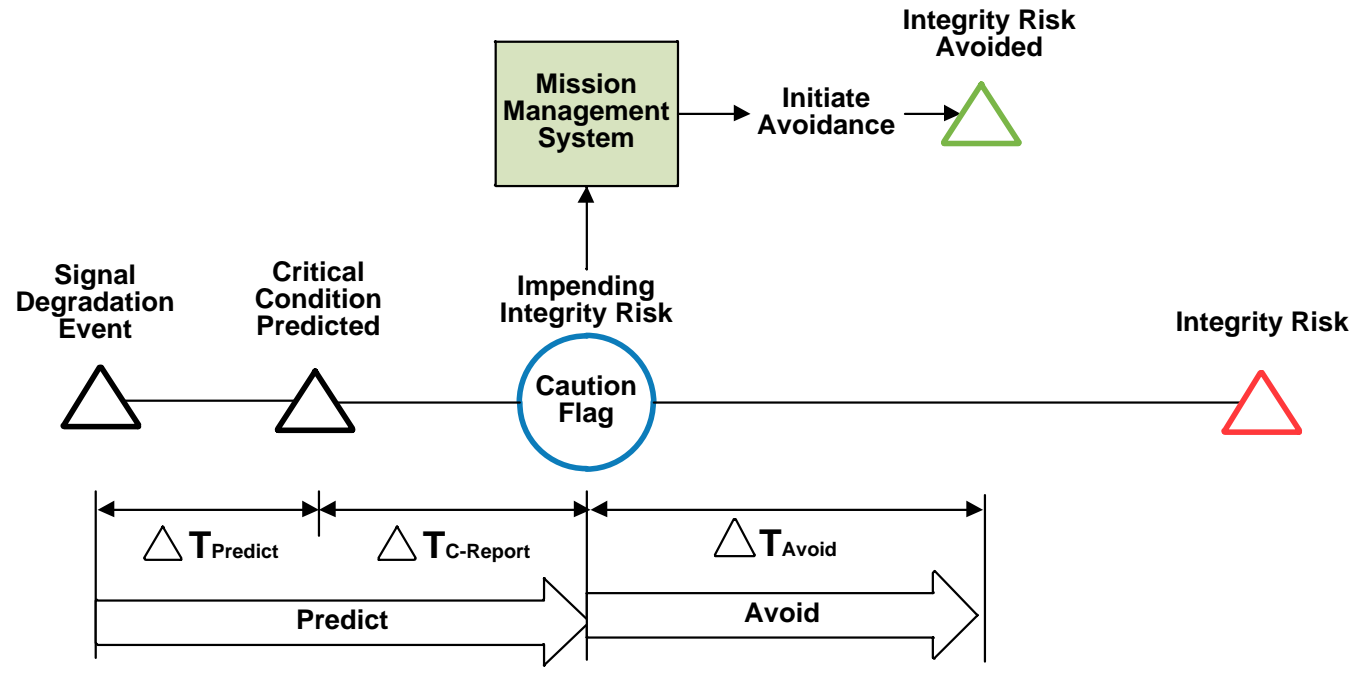

Prediction-Avoidance

Fig. 4 Prediction-avoidance capability of the integrity augmentation system

An integrity augmentation system for a ground vehicle navigating the airport environment (or any urban environment) would have to account for the unpredictability of masking and multipath in such scenarios. Lowering of system accuracy can happen in two ways as described in [8] in: misleading information from the system is not detected, or in the event that such a fault is detected, the user (remote operator) is not informed of it within an acceptable time limit, referred to as Time-To-Alert (TTA). TTA can be defined as the allowable time-window between an integrity risk being detected, and the user being alerted. The system predicts potential sources of signal degradation, which in the case of GNSS navigation would chiefly be multipath, antenna masking and signal propagation losses.
The time required to predict a potential error source is denoted by $\Delta \mathrm{T}_{\text {Predict }} . \Delta \mathrm{T}_{\mathrm{C} \text {-report }}$ denotes the time required to report the potential problem to the mission planner. The reporting is accomplished by means of a caution flag that indicates an imminent malfunction. The mission planner would then initiate avoidance action to avoid a loss of positioning accuracy beyond a threshold that compromises navigation safety. This may involve actions like generating alternative trajectories[36], reducing weightage on GNSS measurements and giving precedence to alternative sensors such as INS or wheel speed sensors. The time taken to execute this is denoted by $\Delta \mathrm{T}_{\text {avoid }}$, and depends on the choice of preventive action. The reaction-correction capability of the integrity augmentation system is illustrated in Fig. 5. 
This is the author pre-publication version. This paper does not include the changes arising from the revision, formatting and publishing processes. The final version that should be used for referencing is:

S. Bijjahalli, S. Ramasamy and R. Sabatini, "A Novel Vehicle-Based GNSS Integrity Augmentation System for Autonomous Airport Surface Operations", Journal of Intelligent \& Robotic Systems, pp. 1-25, 2017. DOI: 10.1007/s10846-017-0479-8

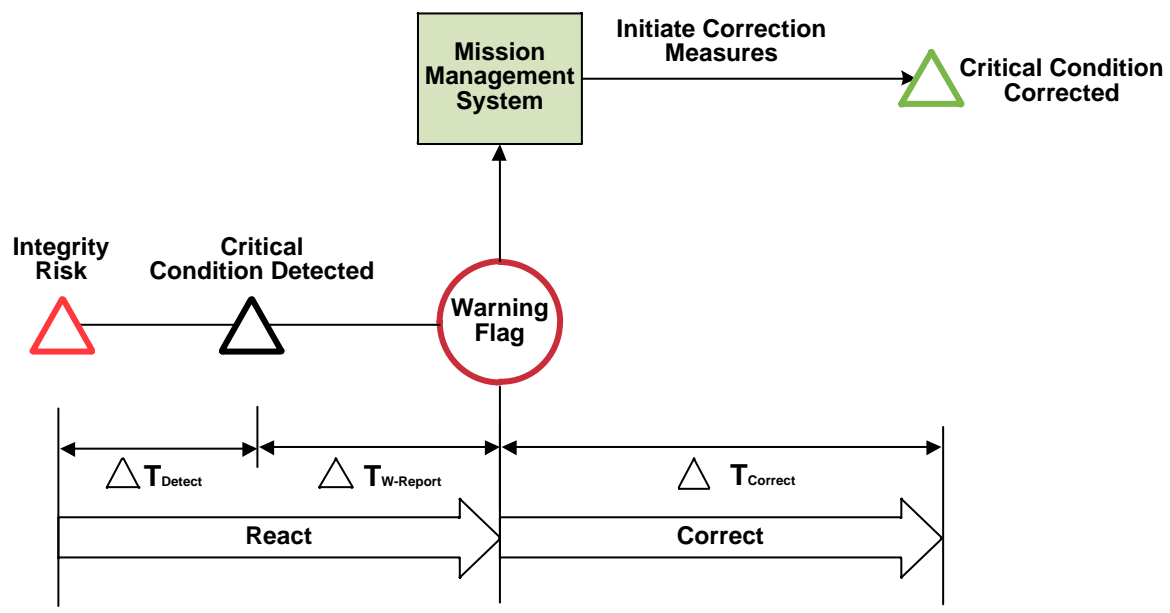

Reaction-Correction

Fig. 5 Reaction-correction capability of the integrity augmentation system

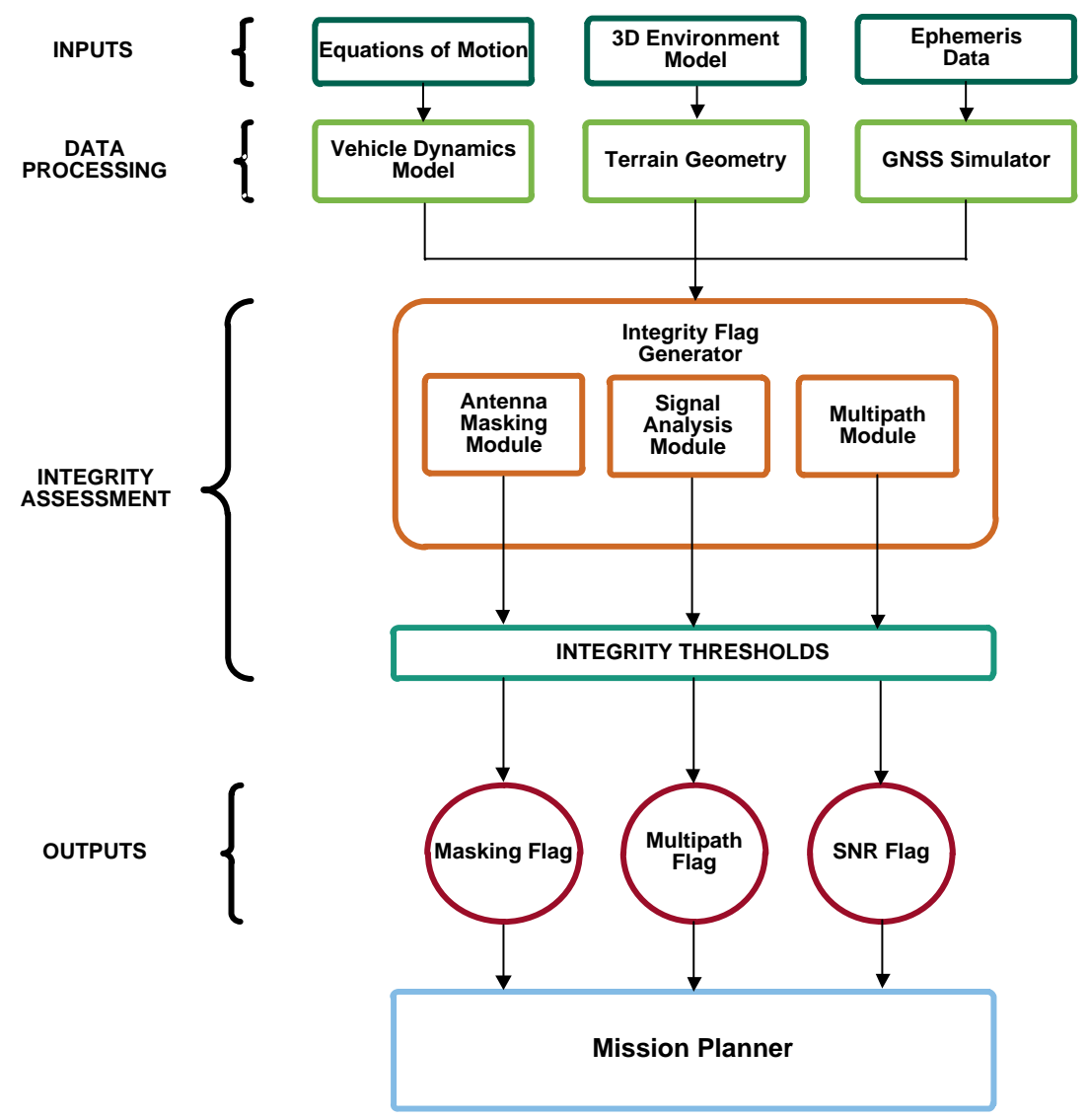

Fig. 6 Integrity augmentation system modules and data flow 
This is the author pre-publication version. This paper does not include the changes arising from the revision, formatting and publishing processes. The final version that should be used for referencing is:

S. Bijjahalli, S. Ramasamy and R. Sabatini, "A Novel Vehicle-Based GNSS Integrity Augmentation System for Autonomous Airport Surface Operations", Journal of Intelligent \& Robotic Systems, pp. 1-25, 2017. DOI: 10.1007/s10846-017-0479-8

The duration for which the caution and warning flags are raised to the autonomous mission planner is termed as Time-to-Caution (TTC) and Time-toWarning (TTW) respectively. The predictionavoidance model holds true when $\Delta \mathrm{T}_{\text {avoid }}<\mathrm{TTC}$. If this condition is violated, system integrity would be compromised making it necessary for reactivecorrection to be initiated. Implementation of the alerting mechanism is achieved through a module that monitors the data provided by the GNSS receiver, termed as the Integrity Flag Generator (IFG). The IFG is illustrated in Fig. 6, along with its respective inputs and outputs.

\section{Sources of GNSS Accuracy Degradation}

This section models the sources of GNSS signal degradations and their effects on the estimated vehicle position. GNSS error sources are broadly divided into multipath induced effects and atmospheric errors classified according to the cause of the degradation as:
- Multipath induced error

- Multipath delay;

- Multipath signal attenuation.

- Atmospheric error

- Ionospheric delay;

- Tropospheric delay;

- Tropospheric scintillation.

The causes of GNSS signal degradation are shown in Fig. 7. The error in GNSS positioning is a function of pseudorange error and the geometry of the satellite constellation at the time of measuring the pseudorange and is given by [18]:

$$
(\text { GPS PVA solution })=(G F) \times(U E R E)
$$

where $G F$ is the Geometry Factor associated with the geometrical configuration of the satellite constellation at the time of measurement, and UERE is the UserEquivalent Range Error, defined as the statistical aggregate of errors associated with each satellite. Neglecting clock biases, the components that comprise UERE are described in [18] and are briefly enumerated in the following section.

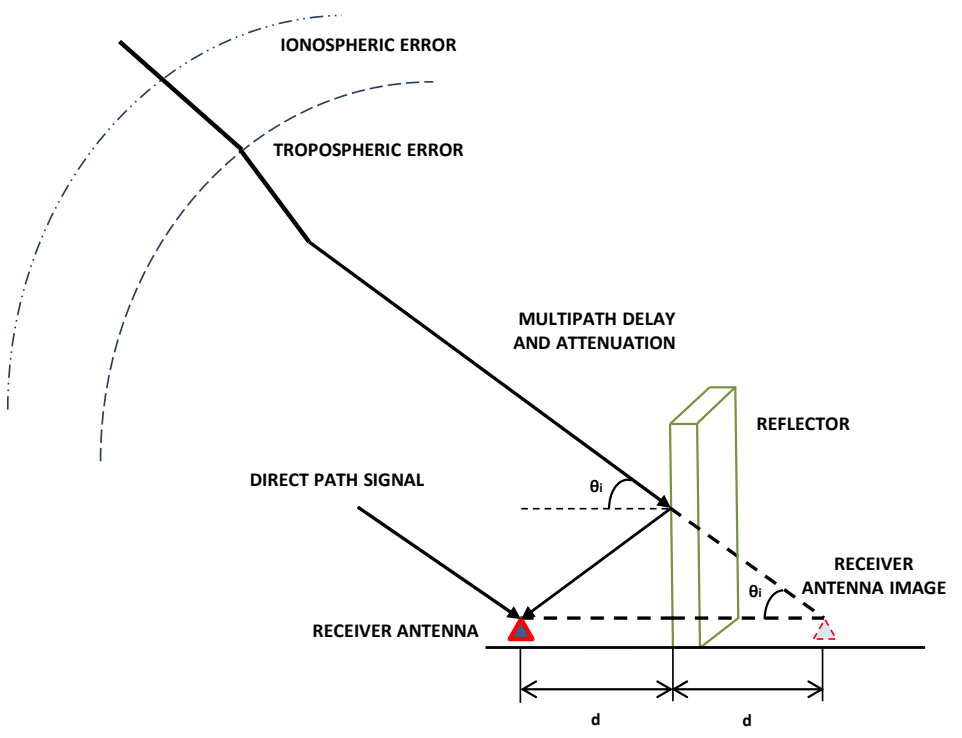

Fig. 7 Causes of GNSS signal degradations. 
This is the author pre-publication version. This paper does not include the changes arising from the revision, formatting and publishing processes. The final version that should be used for referencing is:

S. Bijjahalli, S. Ramasamy and R. Sabatini, "A Novel Vehicle-Based GNSS Integrity Augmentation System for Autonomous Airport Surface Operations", Journal of Intelligent \& Robotic Systems, pp. 1-25, 2017. DOI: 10.1007/s10846-017-0479-8

\subsection{Multipath induced error}

GNSS signal multipath induces error in the carrierphase measured at the receiver. Multipath phase error is a function of the phase shift of the multipath ray, which in turn is a function of the path length difference between the direct and multipath rays. The path length difference is purely dependent on the geometrical relationships between the satellite, reflecting surface and the receiver antenna. In order to verify if multipath exists for a given satellite, reflector and receiver configuration, and to compute the corresponding path-length difference, the receiver image technique[37] as illustrated in Fig. 8 is applied as follows:

- Compute the position of image of the receiver antenna in the reflector;

- Compute the line vector from satellite to image;

- Inspect whether the line vector intersects the reflector. If such an intersection exists, a multipath signal exists in addition to the direct-path signal, and the parameters that describe the multipath signal (delay and phase-shift) are computed.

The ray-tracing method described herein assumes that the GNSS signal is a plane wave, an assumption that is valid owing to the large separation between the source (satellite) and the reflecting surface. A key advantage of the ray-tracing method over existing empirical models [38,39] of signal propagation in urban environments is the ability to deterministically model local signal-terrain interactions in the vicinity of the receiver antenna. The adapted method has been validated in [37,40] through comparison of simulated multipath error with errors in real GNSS signal measurement campaigns. Three experiments were conducted using known reflector geometry and material properties, and the multipath errors were isolated. The experimental results closely matched the multipath error predicted using the ray-tracing model proposed in this study. The variation between the experimental and simulated multipath phase-error was in the range of $\approx 3 \mathrm{~mm}$, which was attributed to measurement noise. This level of noise is in agreement with the expected noise for most modern receivers [41]. Referring to Fig. 8, the algorithm to identify the existence of a multipath GNSS signal for a given satellite-reflector-receiver configuration is as follows:

The location of the satellite (transmitter) is given as:

$$
S=\left[\begin{array}{lll}
x_{s} & y_{s} & z_{s}
\end{array}\right]
$$

The location of the GNSS receiver antenna is given as:

$$
R=\left[\begin{array}{lll}
x_{r} & y_{r} & z_{r}
\end{array}\right]
$$

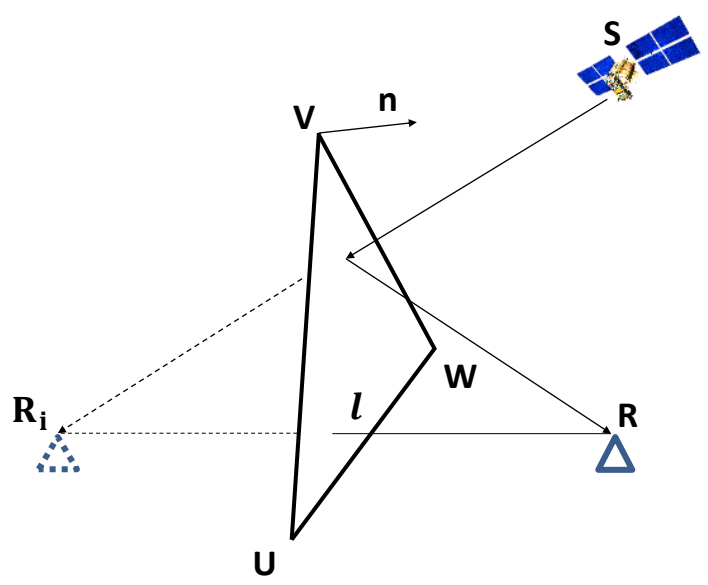

Fig. 8 Multipath ray tracing algorithm

The coordinates of the vertices are known vectors denoted by $U, V, W . n$ is the normal vector to the plane. The shortest line of approach from the receiver antenna $R$ to the reflector is obtained. This line intersects the plane of the reflector at point $l$. The shortest line of approach is quantified by the parameter $t$ given by:

$$
t=\frac{(V-R) \cdot n}{\operatorname{norm}(n)}
$$

The point $l$ is computed as:

$$
l=S+t n
$$

1. Determine the coordinates of the receiver antenna image $R_{i}$, or the reflection of the receiver antenna about the reflector plane by:

$$
R_{i}=R+2(l-R)
$$

2. Check whether the line from $S$ to $R_{i}$ intersects the reflector plane. If so, determine the coordinates of the 
This is the author pre-publication version. This paper does not include the changes arising from the revision, formatting and publishing processes. The final version that should be used for referencing is:

S. Bijjahalli, S. Ramasamy and R. Sabatini, "A Novel Vehicle-Based GNSS Integrity Augmentation System for Autonomous Airport Surface Operations", Journal of Intelligent \& Robotic Systems, pp. 1-25, 2017. DOI: 10.1007/s10846-017-0479-8

intersection point. Referring to Fig. 9, given a plane $\mathcal{P}$, and a line segment described by the parametric equation $P(t)=P_{0}+t\left(P_{L}-P_{0}\right)$, where $P_{0}$ and $P_{L}$ are the start and end-points of the segment (the end point is below the plane $\mathcal{P}$ in Fig. 9), the intersection point is at $P_{I}=P\left(r_{I}\right)$, where the parameter value $r_{I}$ is calculated by:

$$
r_{I}=\frac{n \cdot\left(V-P_{0}\right)}{n \cdot\left(P_{L}-P_{0}\right)}
$$

where $V$ is a point on the plane.

3. After determining the intersection point $P_{I}$ in Step 2 , determine whether the intersection point lies within the reflector. This is performed using an adaptation of [42] as illustrated in Fig. 9.

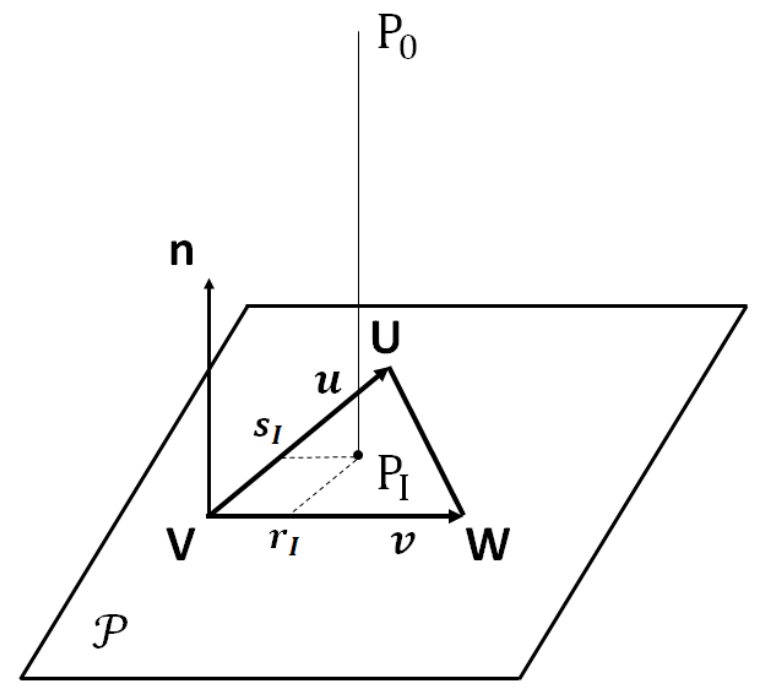

Fig. 9 Intersection of a line and a triangular plane

The intersection point $P_{I}$ of line $S R_{i}$ with the plane $\mathcal{P}$ of the triangle with vertices $U, V$ and $W$ is expressed in parametric coordinates $r_{I}$ and $s_{I}$ given by:

$$
\begin{gathered}
r_{I}=\frac{(u \cdot v)(a \cdot u)-(u \cdot u)(w \cdot v)}{(u \cdot v)^{2}-(u \cdot u)(v \cdot v)} \\
s_{I}=\frac{(u \cdot v)(a \cdot v)-(v \cdot v)(w \cdot u)}{(u \cdot v)^{2}-(u \cdot u)(v \cdot v)}
\end{gathered}
$$

where $a=P_{I}-V$. The following conditions are verified to determine if $P_{I}$ lies within the triangle:

$$
\begin{aligned}
& r \geq 0 \\
& s \geq 0
\end{aligned}
$$

$$
r+s=1
$$

An intersection of $S R_{i}$ with the reflector indicates that a multipath signal exists for the given satellitereflector-receiver configuration. A single direct path signal and a single multipath signal arriving at the receiver antenna is modelled as in [18] and is characterized by amplitude, delay and phase parameters given by:

$$
\begin{gathered}
r(t)=\alpha_{0} e^{-j \widetilde{\phi}_{0}}\left[x\left(t-\tau_{0}\right)+\right. \\
\left.\tilde{\alpha}_{1} e^{-j \widetilde{\phi}_{1}} x\left(t-\tau_{0}-\tilde{\tau}_{1}\right)\right]
\end{gathered}
$$

where $\alpha_{0}$ and $\tau_{0}$ are the amplitude and signal propagation time of the direct-path ray respectively. $\alpha 1$ and $\tau 1$ are the amplitude and signal propagation time of the multipath ray. $\tilde{\phi}_{1}$ and $\tilde{\tau}_{1}$ are the phase and excess delay of the multipath signal with respect to the direct path signal respectively. $\tilde{\alpha}_{1}$ is the Multipath-to-Direct ratio (MDR) of amplitudes $\alpha_{1} / \alpha_{0} \cdot x(t)$ is the complex envelope of the transmitted signal. The oscillator in the receiver generates an identical reference signal given by $e^{-j \theta} x(t)$ and the signal is correlated against the received composite (direct plus multipath) signal to track the received information. The correlation yields the below expression:

$$
\begin{gathered}
\bar{\lambda}(\tau)=\alpha_{0} e^{-j\left(\widetilde{\phi}_{0}-\theta\right)}\left[R_{x}\left(t-\tau_{0}\right)+\right. \\
\left.\tilde{\alpha}_{1} e^{-j \widetilde{\phi}_{1}} R_{x}\left(t-\tau_{0}-\tilde{\tau}_{1}\right)\right]
\end{gathered}
$$

The error in carrier-phase measurement due to the presence of the multipath signal is given as:

$$
\psi=\tan ^{-1}\left[\frac{\widetilde{\alpha}_{1} \sin \widetilde{\phi}_{1} R_{x}\left(t-\tau_{0}-\tilde{\tau}_{1}\right)}{R_{x}\left(t-\tau_{0}\right)+\widetilde{\alpha}_{1} \cos \widetilde{\phi}_{1} R_{x}\left(t-\tau_{0}-\tilde{\tau}_{1}\right)}\right]
$$

The reader is directed to [18] for the derivation of equation 15. $\psi$ is the error in carrier-phase induced by the multipath signal. $R$ is the PRN code correlation function, which is dependent on the time delay $\tau$ of the reflected (multipath) signal relative to the direct path signal and is given by:

$$
\begin{array}{rlrl}
R(\tau) & =1-\frac{|\tau|}{T}, & & \text { for }|\tau| \leq T \\
& =0 & \text { for }|\tau|>T
\end{array}
$$

where $T$ is the PRN code bit (chip) period. If the excess multipath delay $\tilde{\tau}_{1}$ is very small, then equation 
This is the author pre-publication version. This paper does not include the changes arising from the revision, formatting and publishing processes. The final version that should be used for referencing is:

S. Bijjahalli, S. Ramasamy and R. Sabatini, "A Novel Vehicle-Based GNSS Integrity Augmentation System for Autonomous Airport Surface Operations", Journal of Intelligent \& Robotic Systems, pp. 1-25, 2017. DOI: 10.1007/s10846-017-0479-8

15 reduces to:

$$
\psi=\tan ^{-1}\left[\frac{\widetilde{\alpha}_{1} \sin \widetilde{\phi}_{1}}{1+\widetilde{\alpha}_{1} \cos \widetilde{\phi}_{1}}\right]
$$

A similar model has been used to quantify multipath phase error in [37]. The phase shift $\tilde{\phi}_{1}$ of the multipath ray (relative to the direct path ray) is directly proportional to the difference in path lengths between the direct path and multipath rays and is given by:

$$
\tilde{\phi}_{1}=\frac{L_{m}-L_{d}}{\lambda}
$$

where $L_{m}$ and $L_{d}$ are the path lengths of the multipath-signal and the direct signal respectively. The attenuation $\tilde{\alpha}$ of the signal power after being reflected from a surface adversely impacts the signal tracking capability of the receiver, and is a function of the following factors : The relative geometry between the receiver antenna and the reflector, permittivity and polarizing effect of the reflector, and gains of the satellite and receiver antennae. The relationship between the amplitudes, phases and polarization of transmitted and reflected waves is described by the Fresnel equations [43]. If an incoming right-hand circularly polarized signal (GNSS signal) is incident on an interface separating two media at an incidence angle $\theta_{i}$, the signal is partially transmitted through the interface, and partially reflected back into medium 1 . This behaviour can be described in terms of the electric permittivity $\varepsilon$, magnetic permeability $\mu$, refractive index, $\eta$ of the two media. The power of the reflected signal is dependent on the Fresnel reflection coefficient $\rho$. The Fresnel reflection coefficient can be resolved into perpendicular and parallel components as given by Kraus, Fleisch [44]:

$$
\begin{gathered}
\rho_{\perp}=\frac{\cos \theta_{i^{-}} \sqrt{\varepsilon_{2} / \varepsilon_{1}-\sin ^{2} \theta_{i}}}{\cos \theta_{i}+\sqrt{\varepsilon_{2} / \varepsilon_{1}-\sin ^{2} \theta_{i}}} \\
\rho_{\|}=\frac{-\left({ }^{\varepsilon_{2}} / \varepsilon_{1}\right) \cos \theta_{i}+\sqrt{\varepsilon_{2} / \varepsilon_{1}-\sin ^{2} \theta_{i}}}{\left({ }^{\varepsilon_{2}} / \varepsilon_{1}\right) \cos \theta_{i}+\sqrt{\varepsilon_{2} / \varepsilon_{1}-\sin ^{2} \theta_{i}}}
\end{gathered}
$$

where $\rho_{\perp}$ and $\rho_{\|}$are the perpendicular and parallel Fresnel coefficients respectively. The subscripts indicate the medium. For a critical value of $\theta_{i}$ known

as the Brewster angle or polarizing angle, only perpendicular components will be produced after reflection. If the incidence angles are below this critical value, the total Fresnel reflection coefficient, $\rho$ is given by:

$$
\rho=\rho_{\perp} \sin ^{2} \theta_{i}+\rho_{\|} \cos ^{2} \theta_{i}
$$

The fact that the direct path ray and multipath ray arrive at different angles of incidence at the antenna leads to different antenna gains for each signal, the effect of which can be quantified using the antenna gain ratio given by:

$$
\eta_{a}=\frac{G_{d}}{G_{m}}
$$

where $G_{d}$ and $G_{m}$ are the antenna gains of the direct and multipath signals respectively. Given equations 22 and 23 , and assuming a polarization factor $\mathrm{F}=1$, the attenuation factor $\alpha$ of a multipath ray is computed as given in [37]:

$$
\alpha=\rho F \eta_{a}
$$

Given $\alpha$, the multipath induced carrier-phase error in equation 18 is computed. This error translates to an error in receiver position estimation.

\subsection{Calculation of position error due to multipath}

The multipath phase error in Equation 18 biases the satellite-to-receiver antenna range estimate and its effect can be exacerbated by the relative geometry between the satellites and the receiver. The error in the position estimate of the GNSS solution $d x$ can be related to the error in the pseudorange measurements $d \rho$ as given in [18] by:

$$
d x=K d \rho
$$

The subsequent ranging error (between the satellite and receiver) is the product of the carrier-phase error $\psi$ and wavelength of the signal $\lambda$ and is given by:

$$
d \rho=\psi \lambda
$$

$K$ is a function of the satellite constellation geometry that amplifies the range-error to a position-error, given by:

$$
K=\left[\left(H^{T} H\right)^{-1} H^{T}\right]
$$


This is the author pre-publication version. This paper does not include the changes arising from the revision, formatting and publishing processes. The final version that should be used for referencing is:

S. Bijjahalli, S. Ramasamy and R. Sabatini, "A Novel Vehicle-Based GNSS Integrity Augmentation System for Autonomous Airport Surface Operations", Journal of Intelligent \& Robotic Systems, pp. 1-25, 2017. DOI: 10.1007/s10846-017-0479-8

where $H$ is the $n \times 4$ matrix of the components of unit vectors pointing from the receiver location to the satellites ( $n=$ total number of satellites) given by:

$$
H=\left[\begin{array}{cccc}
a_{x 1} & a_{y 1} & a_{z 1} & 1 \\
\vdots & \vdots & \vdots & \vdots \\
a_{x n} & a_{y n} & a_{z n} & 1
\end{array}\right]
$$

If the LOS unit vectors from the receiver antenna to the satellites are known, equation 26 can be used to compute the error in position due to the error in pseudorange. The geometry of the satellite constellation is quantified in practise by the DOP parameter [18]. The Horizontal Dilution Of Precision (HDOP) is used in this paper to quantify the effect of satellite geometry on the airport surface vehicle's position error.

\subsection{Multipath induced SNR variations}

A single reflection model is presented to determine the lowered signal strength due to multipath. The SNR for a single multipath signal is a function of the direct (LOS) signal amplitude $A_{d}$, the multipath signal amplitude $A_{m}$ and the relative phase shift, $\tilde{\phi}$ [45] given by:

$$
\frac{s}{N_{m}}=A_{c}^{2}=A_{d}^{2}+A_{m}^{2}+2 A_{d} A_{m} \cos \tilde{\phi}
$$

where $S$ is the signal strength, $N_{m}$ represents noise in the signal due to multipath, $A_{c}$ is the amplitude of the composite direct plus multipath signal. The phasor diagram for a single multipath and direct signal is shown in Fig. 10, which illustrates the concept of phase lock loop. The GNSS receiver measures inphase (I) and quadrature-phase (Q) components of the signal to estimate the carrier-phase $\psi_{d}$. The presence of the multipath ray introduces error $\psi$ in the carrierphase measurement modelled as given in equation 18 . Equation 29 is rewritten in terms of the MDR $\alpha$ as:

$$
\frac{s}{N_{m}}=\left(\sqrt{1+\alpha^{2}+2 \alpha \cos \psi}\right) A_{d}
$$

The measured SNR is therefore a scaled version of the amplitude direct signal. When there is no multipath, the parameter $\mathrm{M}$ is equal to 1 , and SNR would be equal to the amplitude of the direct signal (neglecting all other sources of noise).

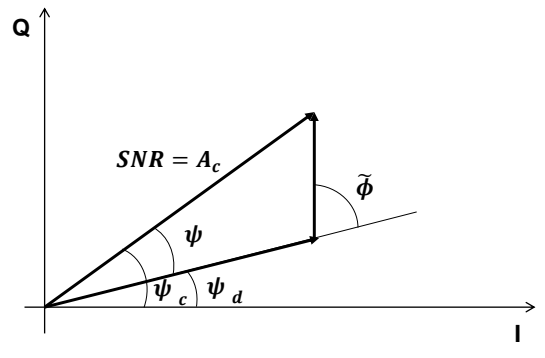

Fig. 10 Phasor diagram for multipath analysis

From [46], SNR is expressed as difference between the transmitted signal power and the noise power :

$$
S N R=\text { Signal Power }(d B)-\text { Noise Power }(d B)(31)
$$

From equations 28 and 29, the noise power due to multipath in $\mathrm{dB}$ is expressed as:

$$
N_{m}=-10 \log M
$$

where

$$
M=\sqrt{1+\alpha^{2}+2 \alpha \cos \psi}
$$

4.4 Atmospheric propagation errors and receiver noise

Signal propagation through the atmosphere introduces errors in the satellite-to-user range estimation and causes signal attenuation, both of which can be modelled for each layer of the atmosphere. The presence of free electrons in the ionosphere affects the index of refraction of the medium through which the signal is travelling [18] which, in turn, affects the wave propagation speed. It is noted in [47] and [25] that ionospheric delay can be broken up into two elements: the first is propagation delay wherein the PRN codes are delayed, and the carrier-phase is advanced. The second is delay due to refraction or bending of the signal, which can be omitted for satellites above an elevation of $15^{\circ}$. Path length differences due to the delays are modelled as:

$$
\begin{gathered}
\Delta S_{\text {iono }, p}=\frac{-40.3 T E C}{f^{2}} \\
\Delta S_{\text {iono }, g}=\frac{40.3 T E C}{f^{2}}
\end{gathered}
$$


This is the author pre-publication version. This paper does not include the changes arising from the revision, formatting and publishing processes. The final version that should be used for referencing is:

S. Bijjahalli, S. Ramasamy and R. Sabatini, "A Novel Vehicle-Based GNSS Integrity Augmentation System for Autonomous Airport Surface Operations", Journal of Intelligent \& Robotic Systems, pp. 1-25, 2017. DOI: 10.1007/s10846-017-0479-8

where $\Delta S_{\text {iono, } p}$ is the signal path length difference due to the phase advance and $\Delta S_{\text {iono, } g}$ is the signal path length difference attributed to the PRN code delay. TEC is the Total Electron Count, which is a measure of electron density along the signal path length, and $f$ is the frequency of the wave. The reader is directed to [18] for a more detailed description of ionospheric modelling. The delay in signal as it propagates through the troposphere is dependent on the refractive index of the medium, which in turn, is a function of the local temperature, pressure and relative humidity. Several models of tropospheric delay are in the literature[48,49]. Equation 36 models the delay as a function of satellite elevation angle [25] :

$$
T D=\operatorname{cosec}\left[E\left(1.4588+0.0029611 N_{s}\right)\right]
$$

where $E$ is the satellite elevation angle and $N_{s}$ is the refraction factor dependant on season and latitude. Signal attenuation occurs in the atmosphere and can be chiefly attributed to the presence of oxygen. The attenuation is modelled as [8]:

$$
A(E) \approx \frac{0.07}{\sin E+0.043} d B
$$

where $A$ is tropospheric attenuation. This expression is relevant for elevation angles between $3^{\circ}$ and $10^{\circ}$. Another source of loss is tropospheric scintillation that varies the refractive index as a function of signal frequency and satellite elevation with respect to the receiver antenna [8] and is given by:

$$
T=0.025 f^{0.58}(\csc E)^{-0.85} d B
$$

where $f$ is the signal frequency $(\mathrm{Hz})$ and $\mathrm{E}$ is the elevation angle in radians. The tropospheric attenuation and scintillation in equations 37 and 38 are combined into a single term $L_{a t m}$, which represents signal loss due to propagation through the atmosphere. The variations in signal power during the propagation of the signal is commonly parameterized by the carrier-to-noise density ratio $\left(C / N_{0}\right)$ in $\mathrm{dB}-\mathrm{Hz}$ adapted from [11]:

$$
\begin{gathered}
C / N_{0}=E I R P+G_{t}+G_{r}-L_{f}-L_{a t m} \\
-N_{t}-N_{f}-N_{m}
\end{gathered}
$$

where EIRP is the Equivalent Isotropic Radiated
Power of the transmitted signal. $L_{f}$ and $L_{a t m}$ are the signal losses in free space and the atmosphere respectively. $N_{t}$ and $N_{f}$ are the receiver antenna thermal noise and the receiver front-end noise respectively. $N_{m}$ is the multipath losses modelled previously. $G_{t}$ and $G_{r}$ are the satellite and receiver antenna gains respectively. Free space propagation loss is given by[50]:

$$
L_{f}=20 \log _{10}\left(\frac{4 \pi d}{\lambda}\right)
$$

where $d$ is the Euclidean distance between the transmitter and receiver antennas and $\lambda$ is the wavelength of the transmitted signal. The thermal noise generated by the antenna is a function of antenna temperature given by:

$$
N_{t}=10 \log _{10}\left(k T_{a} B W\right)
$$

where $B W$ represents the system bandwidth in $\mathrm{Hz}$. The satellite antenna gain $G_{t}$ and receiver antenna gain $G_{r}$ are approximated by [8]:

$$
G_{t}=2.5413 \sin E-2.5413
$$

$$
G_{r}=9.8756 \sin E-4.7567
$$

Equation 39 is used to quantify variations in signal strength owing to the errors introduced during signal propagation, which then forms the basis for setting threshold values or integrity thresholds for the integrity augmentation system.

\section{Integrity Flag Thresholds}

This section describes the procedure followed in determining integrity thresholds based on the GNSS signal degradation models presented in Section 4. Similar thresholds for antenna masking and propagation losses have been introduced [34]. Prior research on GNSS integrity augmentation for aerial navigation assigned thresholds for multipath detection based on the Early Late Phase (ELP) observable originally proposed in [51]. One of the key contributions of this research is the methodology followed in assigning the thresholds for the multipath-induced errors based on the multipath 
This is the author pre-publication version. This paper does not include the changes arising from the revision, formatting and publishing processes. The final version that should be used for referencing is:

S. Bijjahalli, S. Ramasamy and R. Sabatini, "A Novel Vehicle-Based GNSS Integrity Augmentation System for Autonomous Airport Surface Operations", Journal of Intelligent \& Robotic Systems, pp. 1-25, 2017. DOI: 10.1007/s10846-017-0479-8

models described earlier.

\subsection{Thresholds for multipath induced errors}

Given the locations of reflective surfaces in the environment that can potentially cause multipath, and the satellite constellation geometry, the Estimated Horizontal Error (EHE) of the receiver for each GNSS epoch is computed using the models described in Section 4 in an initial data gathering simulation. For all subsequent autonomous runs of the vehicle along the trajectory, values of EHE that exceed the integrity threshold trigger alerts (caution and warning flags) to the mission management system to initiate avoidance measures. Although GNSS multipath is a very actively researched topic, some alert limits have been proposed and introduced for aerial platform implementations $[8,34,52,53]$ but standardised limits have not been prescribed for autonomous ground vehicles. Therefore, based on a trial-and-error approach (targeting an adequate predictive behaviour for the CIF in the intended airport environments), thresholds of 0.17 metres and 0.2 metres were selected for the CIF and WIF activation respectively.
A minimum of four satellites must be visible to the receiver for computing the receiver position and clock bias. If antenna obscuration by environmental objects leads to a scenario with less than 5 satellites in view, a caution flag will be raised. A warning flag will be generated if this number of visible satellites reduces to below four satellites [54].

\subsection{Thresholds for signal attenuation}

Two sources of signal strength attenuation exist. The first is propagation losses which include free space loss and atmospheric effects, and the second is multipath losses. The effect of these error sources is parameterized using the carrier to noise ratio described in Section 4. Given the power of the satellite transmitted signal, the minimum required signal level for the GNSS receiver [46], and the model of signal strength losses, a measured $C / N_{0}<-25 \mathrm{~dB}$ indicates signal strength below the minimum required signal power level. Caution flags are triggered at $C / N_{0}<25$ $\mathrm{dB}-\mathrm{Hz}$ and warning flags are triggered at $C / N_{0}<24$ $\mathrm{dB}-\mathrm{Hz}$. The assigned thresholds are summarised in Table 1.

\subsection{Antenna masking thresholds}

Table. 1 Caution and warning thresholds for GNSS error sources

\begin{tabular}{|c|c|c|}
\hline Type of alert & Integrity Event & Thresholds \\
\hline \multirow{4}{*}{ Caution Flag } & Masking & When number of visible satellites drops to below 5 \\
\cline { 2 - 3 } & Multipath phase error & When $0.17 \mathrm{~m} \leq$ EHE $<0.2 \mathrm{~m}$ \\
\cline { 2 - 3 } & Signal attenuation & When $C / N_{0}$ drops below $25 \mathrm{~dB}-\mathrm{Hz}$ \\
\hline \multirow{3}{*}{ Warning Flag } & Masking & When number of visible satellites drops to below 4 \\
\cline { 2 - 3 } & Multipath phase error & When EHE $\geq 0.2 \mathrm{~m}$ \\
\cline { 2 - 3 } & Signal attenuation & When $C / N_{0}$ drops below $24 \mathrm{~dB}-\mathrm{Hz}$ \\
\hline
\end{tabular}

\section{Airport Surface Vehicle Platform Model}

A four wheeled vehicle with four Degrees-ofFreedom (4-DoF) is modelled as in $[55,56]$ to describe the kinematics of the vehicle.

The following assumptions are taken into consideration:

- The airport surface vehicle tyres are always in contact with the ground.
- Forces at the contact patches of the tyres can be resolved into components in the vehicles $x y$ plane.

- The vehicle has a sufficiently low centre of gravity and hence any roll can be neglected. The xy-plane is always parallel to the XY-plane of the traversed surface.

- The vehicle is front-wheel steered and the steering angle for both front wheels is the same.

In order to simulate vehicle motion, the World Geodetic System (WGS-84) reference frame and the 
This is the author pre-publication version. This paper does not include the changes arising from the revision, formatting and publishing processes. The final version that should be used for referencing is:

S. Bijjahalli, S. Ramasamy and R. Sabatini, "A Novel Vehicle-Based GNSS Integrity Augmentation System for Autonomous Airport Surface Operations", Journal of Intelligent \& Robotic Systems, pp. 1-25, 2017. DOI: 10.1007/s10846-017-0479-8

vehicle body-fixed frame are introduced with axes $X, Y, Z$ and $x, y, z$ respectively. These coordinate frames are illustrated in Fig. 11.

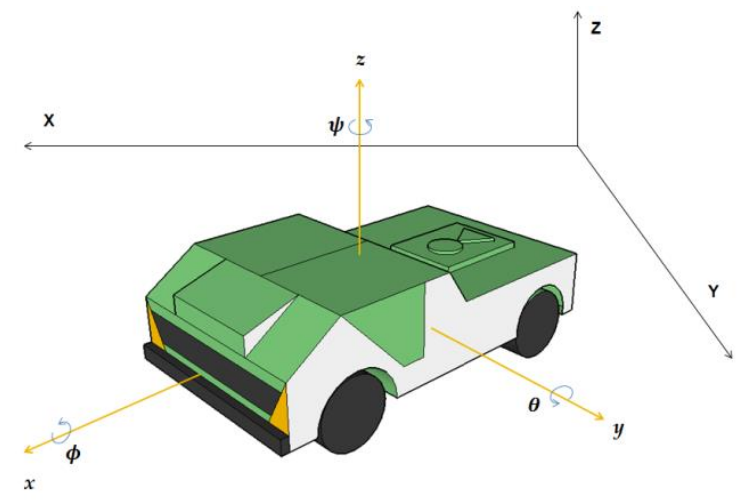

Fig. 11 Coordinate frames

Both reference frames are right-handed cartesian reference frames. The body-fixed reference coordinate frame is defined with the following convention: The $\mathrm{x}$-axis is directed along the longitudinal axis of the vehicle. The y-axis is normal to and directed outwards from the left side of the vehicle. The z-axis is directed upwards completing the right-hand coordinate system. Yaw $(\psi)$, Pitch $(\theta)$ and Roll $(\phi)$ are defined as rotations about the z-axis, $y$-axis and $\mathrm{x}$-axis respectively. The stated assumptions allow the front and rear wheel pairs to be collapsed into a single front wheel and rear wheel. The front wheel has a lateral stiffness (sideslip coefficient) equivalent to twice the lateral stiffness of each individual front wheel and the same condition holds good for the rear wheel. The equations of motion for a vehicle of this type are given by [56], [57]:

$$
\begin{gathered}
\dot{v}_{x}=\frac{F_{x}}{m}+r v_{y} \\
\dot{v}_{y}=\frac{1}{m v_{x}}\left(-a_{1} C_{\alpha f}+a_{2} C_{\alpha r}\right) r- \\
\frac{1}{m v_{x}}\left(C_{\alpha f}+C_{\alpha r}\right) v_{y}+\frac{1}{m} C_{\alpha f} \delta-r v_{x} \\
\dot{r}=\frac{1}{I_{z} v_{x}}\left(-a_{1}{ }^{2} C_{\alpha f}-a_{2}{ }^{2} C_{\alpha r}\right) r-
\end{gathered}
$$


This is the author pre-publication version. This paper does not include the changes arising from the revision, formatting and publishing processes. The final version that should be used for referencing is:

S. Bijjahalli, S. Ramasamy and R. Sabatini, "A Novel Vehicle-Based GNSS Integrity Augmentation System for Autonomous Airport Surface Operations", Journal of Intelligent \& Robotic Systems, pp. 1-25, 2017. DOI: 10.1007/s10846-017-0479-8

\section{Simulation Case Studies}

Four simulation case studies were carried out to test the performance of the designed integrity augmentation system in various airport environments. Trajectories representative of airport surface operations were simulated in two airports including:

- Trajectory (a): San Francisco International Airport (ICAO code: KSFO and IATA: SFO).

- Trajectories (b), (c) and (d): Amsterdam Airport (ICAO code: EHAM and IATA: AMS).

The airports were modelled in Trimble Sketchup ${ }^{\text {TM }}$ environment. The simulated vehicle trajectories are representative of airside cargo trucks that shift luggage from a designated pick-up location to a dropoff point next to the cargo bay of aircraft parked on the apron. Fig. 12 illustrates the airport apron adjacent to terminal two of San Francisco International Airport and Trajectory (a).

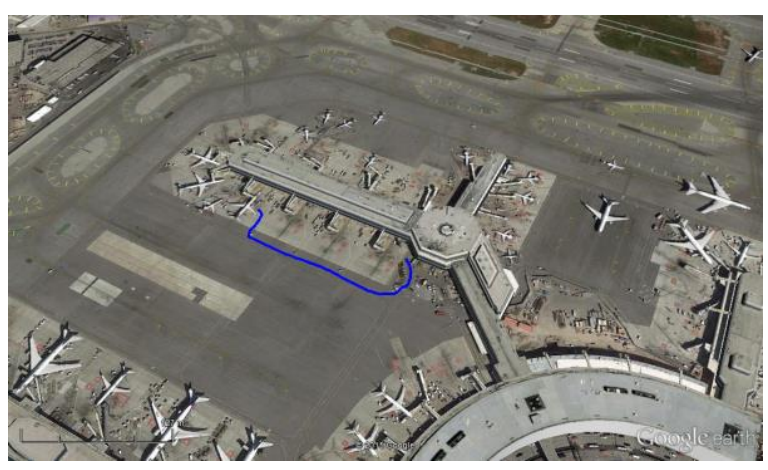

Fig. 12 Simulated scenario at KSFO airport

The measurements of the GNSS receiver mounted on the vehicle are affected by multipath caused by reflections from buildings and aircraft. The multipath introduces error in the carrier-phase measurement and attenuates the signal. The erroneous carrier-phase translates to an error in the computed position that is exacerbated by obstructions or masking of the receiver antenna. Additionally, the signal power is also attenuated by its propagation through the layers of the atmosphere. The purpose of the integrity augmentation system is to detect these sources of degradation and raise alerts to the mission management system in a timely manner. The following integrity augmentation modules of the system architecture are implemented in MATLAB ${ }^{\mathrm{TM}}$ :

- Signal analysis module: Given the satellite elevation and line-of-sight vector between the receiver antenna and satellite, this module computes the carrier-to-noise ratio as modelled in section 4 and raises integrity alerts in the event of atmospheric attenuation;

- Antenna masking module: This module detects blockage of the line-of-sight vector by objects in the environment given the object geometry and locations;

- Multipath module: This module provides phase error, range error and delay due to multipath using the ray tracing algorithm described in section 4.

\subsection{Satellite constellation}

A GPS constellation was simulated in MATLAB ${ }^{\mathrm{TM}}$ by using ephemeris data extracted from a YUMA almanac [58] that can be propagated to give satellite positions in the Earth-Centred Earth-Fixed (ECEF) coordinate system. This enables computation and tracking of LOS vectors between satellites and a given vehicle receiver.

\subsection{Satellite and receiver parameters}

The satellite and receiver parameters including EIRP, receiver antenna temperature and front end noise and bandwidth requirements are based on the link budget analysis presented in [46] and presented in Table 2.

Table. 2 Satellite and receiver parameters

\begin{tabular}{|c|c|}
\hline Parameter & Value \\
\hline EIRP & $26.8 \mathrm{dbW}$ \\
\hline Receiver antenna temperature & $513 \mathrm{~K}$ \\
\hline Receiver front end noise & $2 \mathrm{~dB}$ \\
\hline Carrier frequency & $1575 \mathrm{MHz}$ \\
\hline System bandwidth & $2 \mathrm{MHz}$ \\
\hline
\end{tabular}

7.3 Vehicle dynamics model

In order to generate a vehicle trajectory, the statespace model of vehicle dynamics and the steering controller described earlier was modelled in MATLAB $^{\mathrm{TM}}$ and Simulink. The input to the vehicle 
This is the author pre-publication version. This paper does not include the changes arising from the revision, formatting and publishing processes. The final version that should be used for referencing is:

S. Bijjahalli, S. Ramasamy and R. Sabatini, "A Novel Vehicle-Based GNSS Integrity Augmentation System for Autonomous Airport Surface Operations", Journal of Intelligent \& Robotic Systems, pp. 1-25, 2017. DOI: 10.1007/s10846-017-0479-8

model is the steering angle and the output is the PVA in the local ENU frame.

\subsection{Navigation environment geometry}

The antenna masking and multipath modules require the geometry of the environment as inputs. The reflective surfaces in the navigation scenario are the facades of nearby buildings (airport terminals in this case). The simulation of GNSS signal multipath is restricted to first order reflections from nearby reflective surfaces. Ground echoes can be safely neglected since software defined receivers can be configured to reject signal rays arriving at a low elevation angle (usually $<10^{\circ}$ ). This can also be achieved by using choke ring antennas that can eliminate signals arriving from below the antenna phase centre.

\subsection{Meshing of airport environment}

The constructed airport geometry is converted to a STereoLithography (STL) file representing the airport environments as a set of Cartesian coordinates, which are then meshed into discrete triangular elements that are assumed to be infinitely thin, uniformly dielectric and non-magnetic. In order to represent the airport scenario comprehensively, aircraft CAD models were added to the environment to simulate aircraft on the apron. The procedure adopted can be summarised as follows:

- Create a model of the navigation environment in 3D modelling software.

- Convert the CAD file to a STL file. This meshes the models into triangular elements.

- Import the STL file into MATLAB ${ }^{\mathrm{TM}}$ to extract triangle vertices coordinates. These coordinates are then used in the ray-tracing algorithm to simulate antenna masking and multipath.

This three-step procedure is illustrated in Fig.13. Once the triangular mesh vertex coordinates are obtained and stored in the software compatible matrix form, the antenna masking and multipath influences on the GNSS data as the signal travels along a given path can be determined.
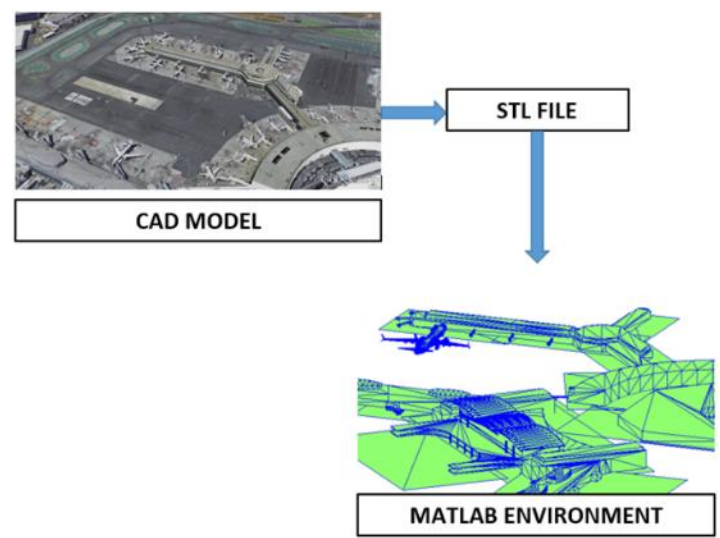

Fig. 13 Navigation environment and geometry meshing

\subsection{Initial planning and data gathering}

The trajectory of the airport surface vehicle as it steers from the terminal to the aircraft and vice versa is depicted in Fig. 14. An initial data gathering and planning stage is performed to calculate multipath induced carrier-phase error, HDOP, amplitude attenuation, and the corresponding position-error for the planned trajectory.

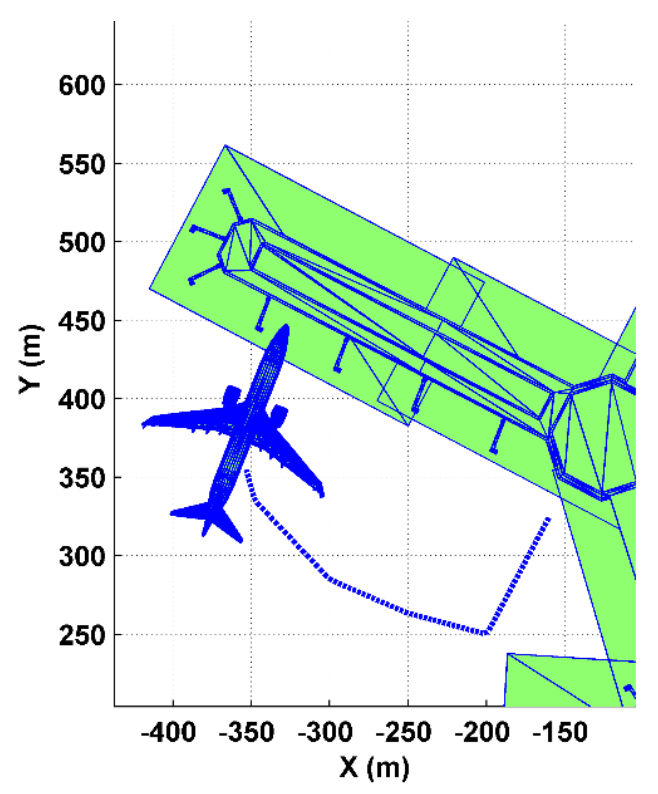

Fig. 14 Simulated trajectory at KSFO airport Trajectory (a) 
This is the author pre-publication version. This paper does not include the changes arising from the revision, formatting and publishing processes. The final version that should be used for referencing is:

S. Bijjahalli, S. Ramasamy and R. Sabatini, "A Novel Vehicle-Based GNSS Integrity Augmentation System for Autonomous Airport Surface Operations", Journal of Intelligent \& Robotic Systems, pp. 1-25, 2017. DOI: 10.1007/s10846-017-0479-8

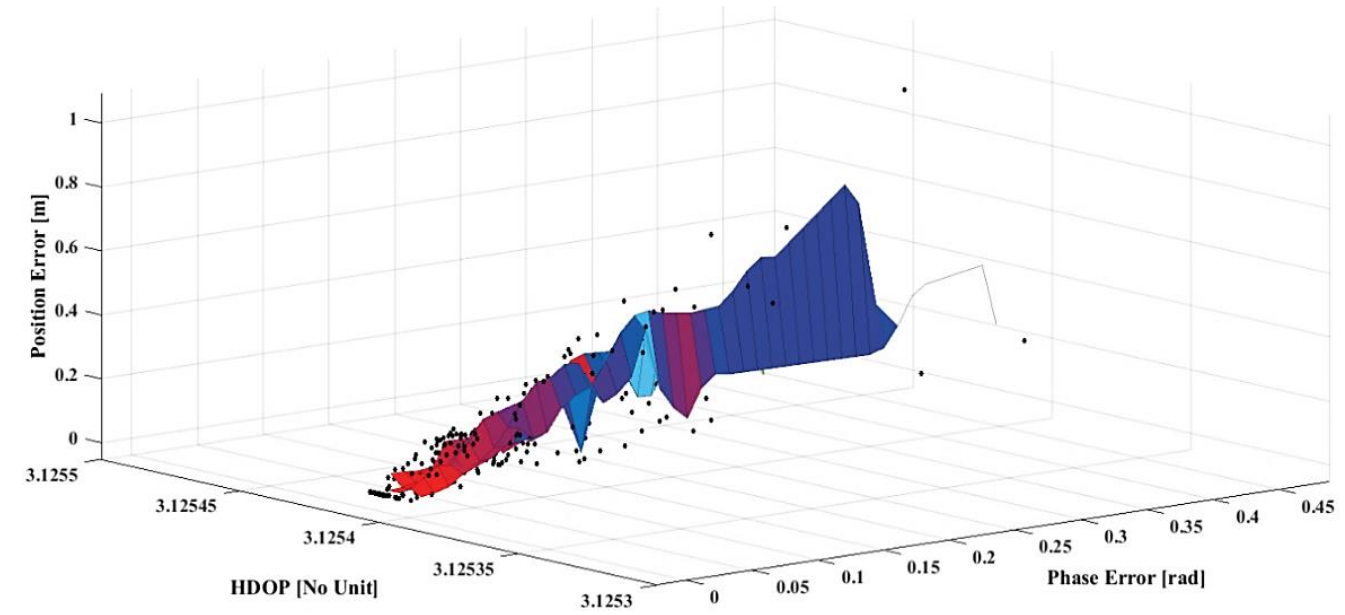

Fig. 15 Surface-fitted model of position error against phase error and HDOP

The integrity thresholds for the trajectory are set as in Table 1, and used for all subsequent autonomous runs along that trajectory. The relationship between carrierphase error, HDOP and position error was described by fitting a surface to the data gathered from the initial simulation. A linear interpolated surface was found to closely fit the data with a high value of the R-square coefficient $(\mathrm{R}$-square $=0.9995)$ as shown in Fig. 15 . The fitted surface was then used during subsequent autonomous runs to infer the EHE in real-time to compare against the assigned threshold. The algorithm implemented to generate masking and multipath flags is illustrated in Fig. 16.

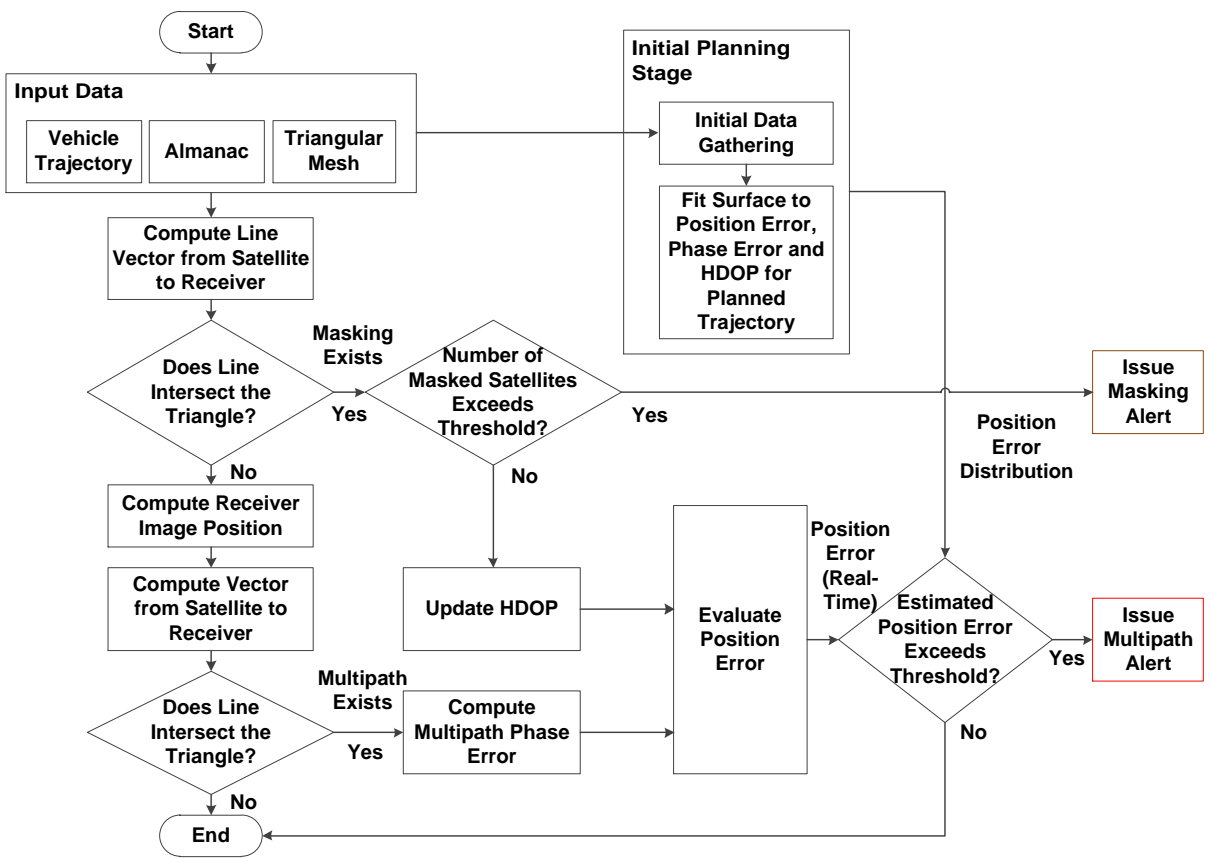

Fig. 16 Algorithm for generating integrity alerts 
This is the author pre-publication version. This paper does not include the changes arising from the revision, formatting and publishing processes. The final version that should be used for referencing is:

S. Bijjahalli, S. Ramasamy and R. Sabatini, "A Novel Vehicle-Based GNSS Integrity Augmentation System for Autonomous Airport Surface Operations", Journal of Intelligent \& Robotic Systems, pp. 1-25, 2017. DOI: 10.1007/s10846-017-0479-8

\subsection{Results}

The performance of the integrity augmentation system is presented for the four simulated trajectories. Generation of alert flags due to multipath phase-error is visualised in Fig. 17. Integrity alerts for antenna masking were raised for only a brief period of time

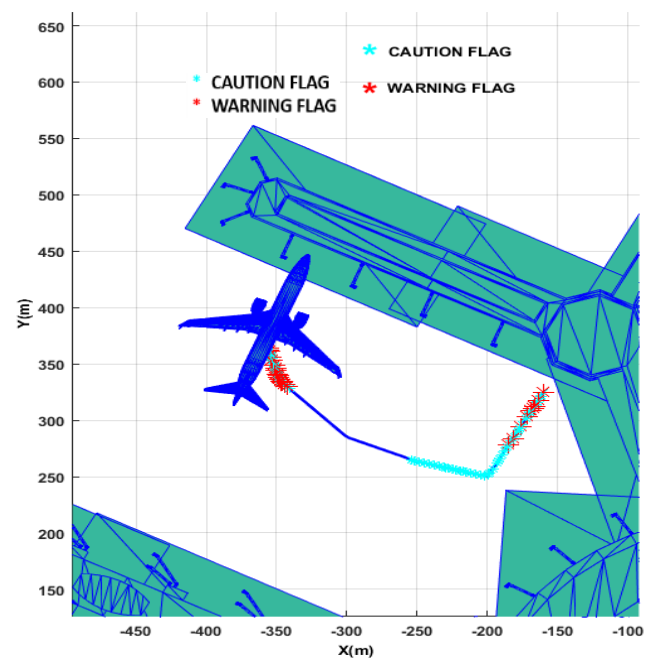

(i)

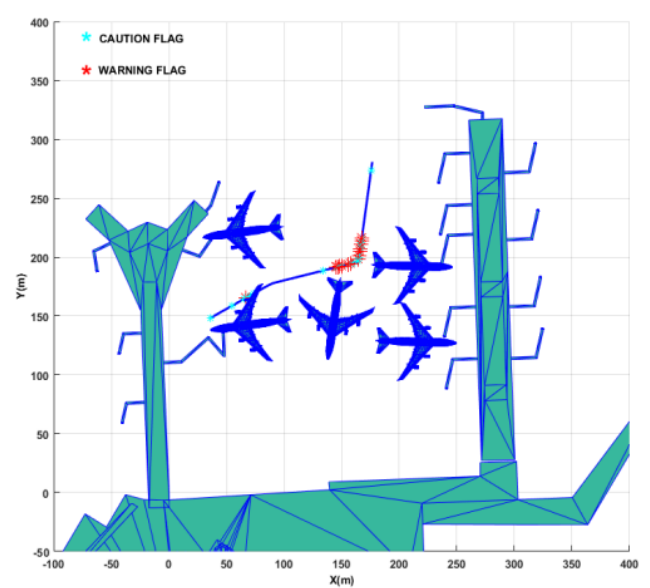

(ii) during trajectory (a): Caution flags were raised in the interval between $t=0 \mathrm{sec} \sim t=10 \mathrm{sec}$, and between $t=314 \mathrm{sec} \sim t=324 \mathrm{sec}$. No warning flags were raised for antenna masking, implying that the number of visible satellites remained above 4 throughout the simulation.

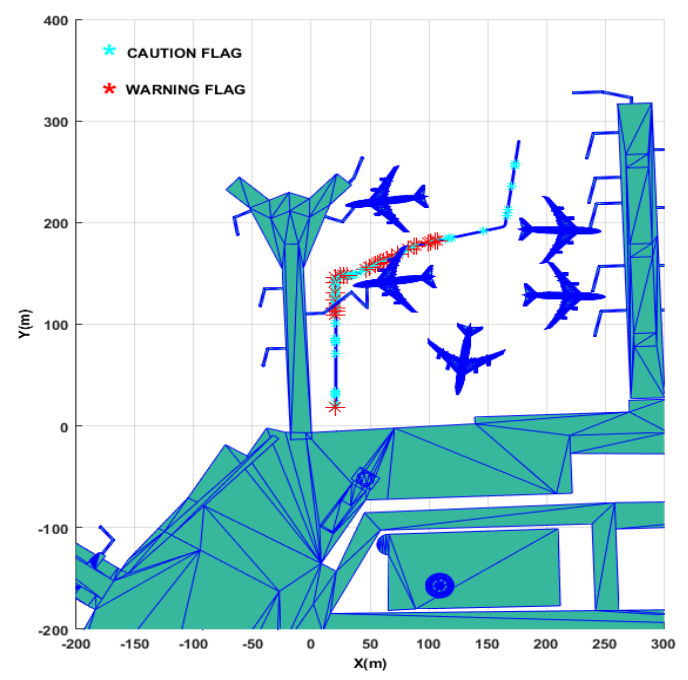

(iii)

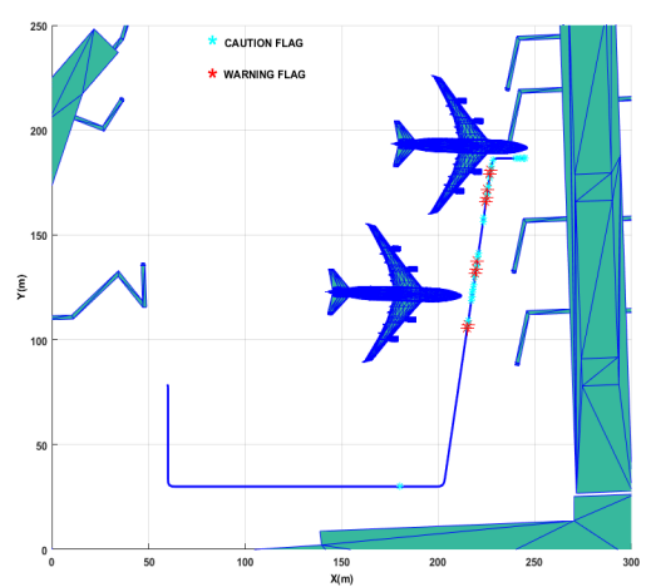

(iv)

Fig. 17 Caution and warning flags for multipath phase-error for (i) Trajectory (a), (ii) Trajectory (b),

(iii) Trajectory (c) and (iv) Trajectory (d) 
This is the author pre-publication version. This paper does not include the changes arising from the revision, formatting and publishing processes. The final version that should be used for referencing is:

S. Bijjahalli, S. Ramasamy and R. Sabatini, "A Novel Vehicle-Based GNSS Integrity Augmentation System for Autonomous Airport Surface Operations", Journal of Intelligent \& Robotic Systems, pp. 1-25, 2017. DOI: 10.1007/s10846-017-0479-8

The results of the simulations show that the designed integrity monitoring system is capable of raising alerts predictively in the presence of multipath induced phase error, antenna masking and lowered signal strength. As expected, the number of flags raised was higher when the vehicle trajectory was closer to the buildings and aircraft. The time steps at which the flags are raised for the four simulated trajectories are presented in Table 3.

The validity of the caution flags raised is analysed by determining True Caution Flags (TCF) as defined in [54].

Table. 3 Time steps at which alerts are raised for multipath phase errors

\begin{tabular}{|c|c|c|}
\hline \multicolumn{3}{|r|}{ Multipath Caution and Warning Integrity Flag Epochs } \\
\hline \multirow{2}{*}{$\begin{array}{c}\text { San Francisco } \\
\text { Airport - } \\
\text { Trajectory (a) }\end{array}$} & $\begin{array}{c}\text { Caution } \\
\text { Flag }\end{array}$ & $1 \sim 8,10 \sim 21,24 \sim 28,30 \sim 39,41 \sim 43,46,48 \sim 49,51,53,55,57,59 \sim 61,63 \sim 64,66 \sim 68,133 \sim 151$ \\
\hline & $\begin{array}{c}\text { Warning } \\
\text { Flag }\end{array}$ & $7 \sim 8,12,15 \sim 17,25 \sim 31,34,134 \sim 141,143 \sim 144$ \\
\hline \multirow{2}{*}{$\begin{array}{c}\text { Amsterdam } \\
\text { Airport - } \\
\text { Trajectory (b) }\end{array}$} & $\begin{array}{c}\text { Caution } \\
\text { Flag }\end{array}$ & 94,146 148,153 157,159 165,173 174,178 189,198 202 \\
\hline & $\begin{array}{l}\text { Warning } \\
\text { Flag }\end{array}$ & 198 202 \\
\hline \multirow{2}{*}{$\begin{array}{c}\text { Amsterdam } \\
\text { Airport - } \\
\text { Trajectory (c) }\end{array}$} & $\begin{array}{l}\text { Caution } \\
\text { Flag }\end{array}$ & $1,12,44 \sim 55,58 \sim 60,63 \sim 66,108 \sim 111,117,128$ \\
\hline & $\begin{array}{c}\text { Warning } \\
\text { Flag }\end{array}$ & $45,47,49 \sim 50,52,54,59 \sim 60,64 \sim 66,110$ \\
\hline \multirow{2}{*}{$\begin{array}{c}\text { Amsterdam } \\
\text { Airport - } \\
\text { Trajectory (d) }\end{array}$} & $\begin{array}{l}\text { Caution } \\
\text { Flag }\end{array}$ & $83 \sim 84,87 \sim 88$, , 97 102, 106, 113 117, 121 122, 129 137, 135, 142 145, \\
\hline & $\begin{array}{c}\text { Warning } \\
\text { Flag }\end{array}$ & $116 \sim 117,145$ \\
\hline
\end{tabular}

If a caution flag is followed by a warning flag, this implies the integrity monitoring system has correctly predicted an imminent degradation in accuracy beyond the specified warning threshold and the caution flag is designated as a TCF. Metrics used to measure the system performance are the Detection Rate (DR) given by warning flags preceded by caution flags, and the Caution Detection Rate (CDR) given by [54]:

$$
C D R=\frac{T C F}{T C F+F C F}
$$

The number of TCF, false caution flags (caution flags not followed by warning flags) and warning flags, along with the computed DR for Trajectory (a) are shown in Table 4.
Positioning error due to multipath affects GNSS data integrity in all three simulated trajectories, and is detected with an average DR of 1.0 and CDR of 0.45 when applying the integrity thresholds specified in Table 1. Fig 18 shows integrity alerts raised due to lowered signal strength due to a combination of propagation and multipath losses for SV2, which is at an average elevation of approximately $24^{\circ}$ relative to the receiver antenna for the duration of the simulation. The time series of C/N0 ratio for Trajectory (a) for all satellites in the simulation are shown in Fig. 19, which represents the signal strength and noise levels due to free space loss, atmospheric loss, multipath and receiver noise. 
This is the author pre-publication version. This paper does not include the changes arising from the revision, formatting and publishing processes. The final version that should be used for referencing is:

S. Bijjahalli, S. Ramasamy and R. Sabatini, "A Novel Vehicle-Based GNSS Integrity Augmentation System for Autonomous Airport Surface Operations", Journal of Intelligent \& Robotic Systems, pp. 1-25, 2017. DOI: 10.1007/s10846-017-0479-8

Table. 4 Generation of integrity flags for trajectory (a) - performance analysis

\begin{tabular}{|c|c|c|c|}
\hline \multirow{4}{*}{$\begin{array}{l}\text { Multipath } \\
\text { Phase Error }\end{array}$} & Total number of Caution Flags & 12 & \multirow{4}{*}{$\begin{aligned} \text { Multipath DR } & =1.0 \\
\text { Multipath CDR } & =0.41\end{aligned}$} \\
\hline & Number of True Caution Flags & 5 & \\
\hline & Number of Warning Flags & 7 & \\
\hline & $\begin{array}{c}\text { Number of Warning flags preceded by } \\
\text { Caution Flags }\end{array}$ & 7 & \\
\hline \multirow{4}{*}{ Masking } & Total number of Masking Caution Flags & 1 & \multirow{4}{*}{$\begin{array}{l}\text { Masking DR }=1.0 \\
\text { Masking } \mathrm{CDR}=0\end{array}$} \\
\hline & Number of True Caution Flags & 0 & \\
\hline & Number of Warning Flags & 0 & \\
\hline & $\begin{array}{c}\text { Number of Warning flags preceded by } \\
\text { Caution Flags }\end{array}$ & 0 & \\
\hline \multirow{4}{*}{ Carrier-to-Noise } & Total number of signal loss Caution Flags & 21 & \multirow{4}{*}{$\begin{aligned} \text { Signal Loss DR } & =1.0 \\
\text { Signal Loss CDR } & =0.47\end{aligned}$} \\
\hline & Number of True Caution Flags & 10 & \\
\hline & Number of Warning Flags & 10 & \\
\hline & $\begin{array}{c}\text { Number of Warning flags preceded by } \\
\text { Caution Flags }\end{array}$ & 10 & \\
\hline
\end{tabular}

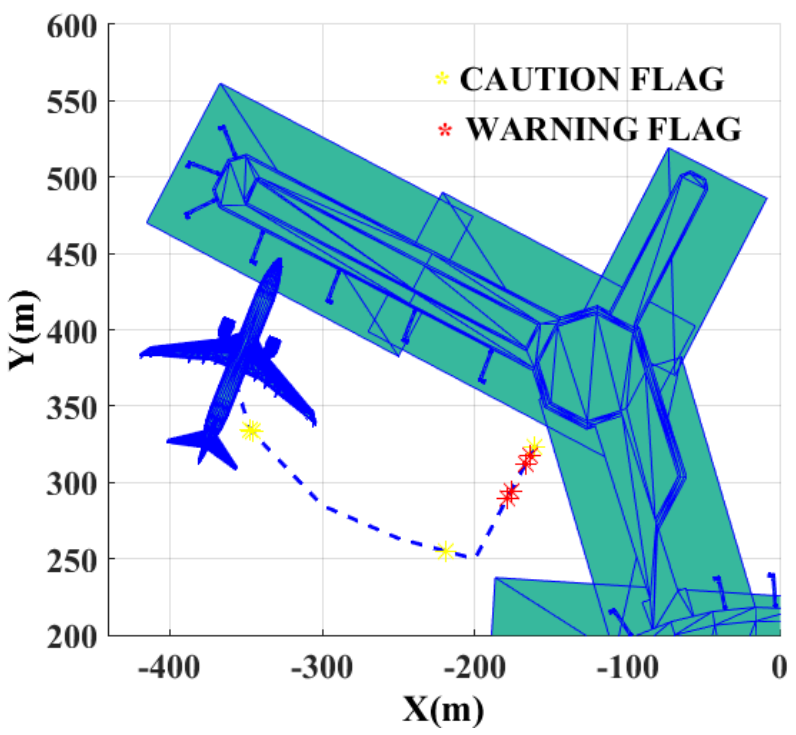

Fig. 18 Integrity alerts for signal attenuation in Trajectory (a) 
This is the author pre-publication version. This paper does not include the changes arising from the revision, formatting and publishing processes. The final version that should be used for referencing is:

S. Bijjahalli, S. Ramasamy and R. Sabatini, "A Novel Vehicle-Based GNSS Integrity Augmentation System for Autonomous Airport Surface Operations", Journal of Intelligent \& Robotic Systems, pp. 1-25, 2017. DOI: 10.1007/s10846-017-0479-8

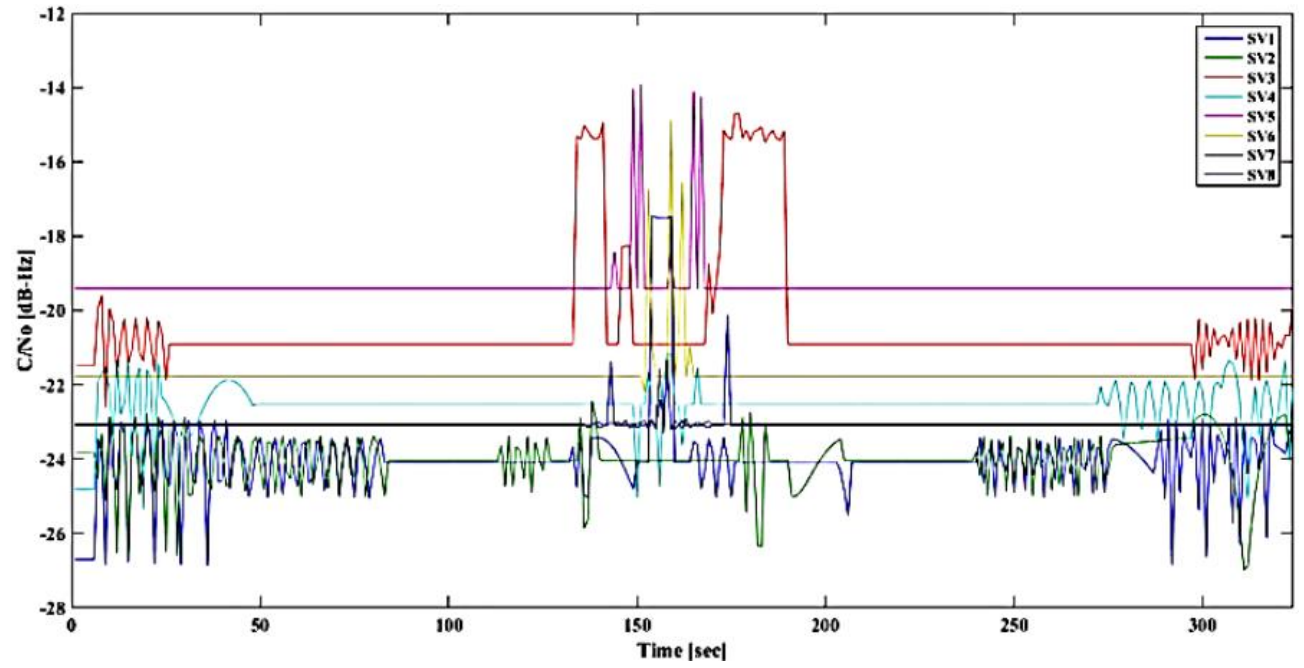

Fig. 19 Carrier-to-noise ratio for all visible satellites - Trajectory (a)

Masking and Signal loss were not major factors affecting GNSS data integrity in trajectories (b) and (c). This can be explained by the fact that visible satellite elevations in these trajectories are in the range of $40^{\circ}-45^{\circ}$.
Subsequently, the high signal strength for these satellites renders them impervious to fluctuations caused by multipath. Antenna masking was found to be an integrity concern in Trajectory (d) as illustrated by the triggering of caution and warning flags in Fig. 20.

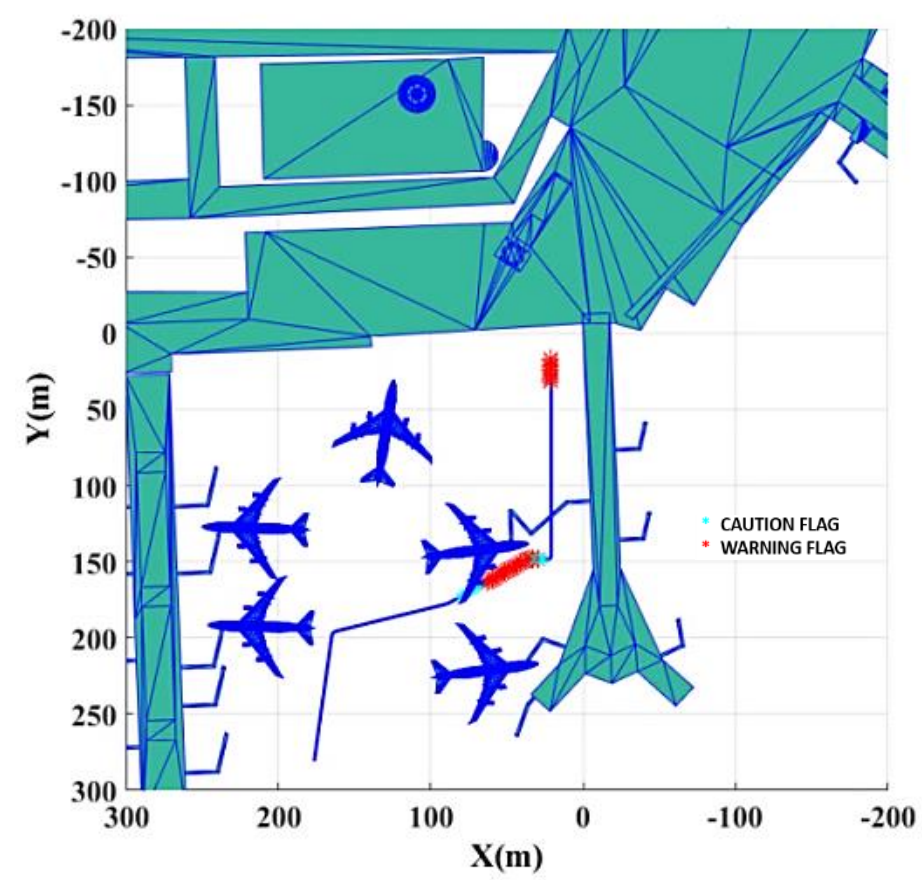

Fig. 20 Caution and warning flags for antenna masking for trajectory 
This is the author pre-publication version. This paper does not include the changes arising from the revision, formatting and publishing processes. The final version that should be used for referencing is:

S. Bijjahalli, S. Ramasamy and R. Sabatini, "A Novel Vehicle-Based GNSS Integrity Augmentation System for Autonomous Airport Surface Operations", Journal of Intelligent \& Robotic Systems, pp. 1-25, 2017. DOI: 10.1007/s10846-017-0479-8

The minimum TTC recorded was 1 second, which represents the predictive capability of the system for the assigned alert thresholds. This performance was found to be worse than the ABIA system developed for aircraft flight operations [34], where a TTC of at least 2 seconds was achieved. However, it must be noted that the alerts in [34] were restricted to errors caused by aircraft manoeuvres and aircraftsatellite relative geometry, which differ from the GNSS errors experienced by ground vehicles in dense airport environments. Additionally, ground vehicles are subject to fewer perturbations, making it feasible to predict trajectories with greater accuracy.

For trajectories (a) and (c), it can be inferred that under the current thresholds, the system is overly sensitive to multipath induced errors in carrierphase measurement, and will lead to an excessive false-alarm rate when implemented on an autonomous system. Similar behavior was observed for the integrity flags raised for signal attenuation in Trajectory (a). Even though all warning flags were preceded by caution flags, a large number of false caution flags were raised. The favorable effect of high satellite elevation angle on signal strength can be inferred for satellites 3 to 8 , all of which are at average elevation angles higher than $30^{\circ}$, and do not approach the assigned integrity thresholds for $C / N_{0}$ in spite of being affected by multipath noise. On the other hand, satellites 1 and 2 are both at elevation angles of approximately $24^{\circ}$, making them susceptible to a loss of receiver tracking owing to the multipath induced variations in signal strength, which subsequently triggers integrity alerts. It is envisaged that if by further fine tuning the alert thresholds, the methodology presented in this paper can be used to design autonomous ground vehicle mission management systems that predict GNSS data losses and pre-emptively compensate for them for all surface vehicle operations.

\section{Conclusion}

The design and implementation of a differential carrier-phase GNSS based Navigation and Guidance System (NGS) for autonomous airport surface vehicle operations was presented. The system was designed to provide trusted autonomy in airport ground operations including cargo-handling, aircraft-towing, passenger transport, etc. The key focus was on modelling GNSS signal degradations affecting the overall system integrity. Based on the identified GNSS signal degradation mechanisms, an integrity monitoring and augmentation system was introduced to produce timely alert flags based on both model-predictive and reactive (observationbased) features. Multipath was found to be the dominating source of integrity degradation owing to its dependence on the complex nature of the airport navigation environment. A ray tracing algorithm was adopted to detect multipath and compute its associated parameters, namely multipath delay, amplitude attenuation and phase error, which served as metrics to indicate integrity degradation. The airport vehicle platform and steering controller was modelled and realistic trajectories were generated to test the integrity augmentation system and its assigned thresholds through simulation. A model of positioning error as a function of multipath parameters and the satellite geometry (unique to the given trajectory and navigation environment) was generated prior to the autonomous run. During the autonomous run, integrity alerts were generated in real-time, from which the following conclusions are deduced:

- The presented integrity augmentation system is capable of early detection of GNSS accuracy and signal degradations due to multipath, masking and attenuation.

- The model was also tested for various other trajectories to determine whether accuracy degradation could be detected as in the initial tested trajectory. A similar detection of GNSS errors leads to the inference that a single model of positioning error for one trajectory and navigation environment, can be used to provide integrity alerts during autonomous runs for all classes of airport ground vehicles.

The multipath model in this study was deterministic in nature and confined to single-order reflections as 
This is the author pre-publication version. This paper does not include the changes arising from the revision, formatting and publishing processes. The final version that should be used for referencing is:

S. Bijjahalli, S. Ramasamy and R. Sabatini, "A Novel Vehicle-Based GNSS Integrity Augmentation System for Autonomous Airport Surface Operations", Journal of Intelligent \& Robotic Systems, pp. 1-25, 2017. DOI: 10.1007/s10846-017-0479-8

a starting point of research. Future research is aimed at extending this concept to multiple reflections and validating the described methodology through conducting real-time experiments. The feasibility of using the presented algorithm to facilitate guidance and trajectory optimization in the presence of GNSS error sources will be explored as a future topic for individual vehicles and fleets.

\section{References}

1. Lacagnina, M.: Defusing the ramp. AeroSafety world 2(5) (2007).

2. McDonald, S.S.: Personal Rapid Transit personal rapid transit (PRT) system and Its Development personal rapid transit (PRT) system development. In: Transportation Technologies for Sustainability. pp. 831-850. Springer, (2013)

3. Spriggs, T.J.: Autonomous vehicle guidance on or near airports. In. Google Patents, (2005)

4. Thrun, S., Montemerlo, M., Dahlkamp, H., Stavens, D., Aron, A., Diebel, J., Fong, P., Gale, J., Halpenny, M., Hoffmann, G.: Stanley: The robot that won the DARPA Grand Challenge. Journal of field Robotics 23(9), 661-692 (2006).

5. O’Connor, M.L.: Carrier-phase differential GPS for automatic control of land vehicles. Stanford University (1997)

6. Bevly, D.M., Rekow, A., Parkinson, B.: Comparison of INS vs. Carrier-Phase DGPS for Attitude Determination in the Control of OffRoad Vehicles. Navigation 47(4), 257-266 (2000).

7. Andrés, S., Daniel, C.: Integrity monitoring applied to the reception of GNSS signals in urban environments. (2012).

8. Sabatini, R., Moore, T., Hill, C.: A New Avionics-Based GNSS Integrity Augmentation System: Part 1-Fundamentals. Journal of Navigation 66(03), 363-384 (2013).

9. Sabatini, R., Moore, T., Hill, C.: Avionics-based integrity augmentation system for mission-and safety-critical GNSS applications. In: proceedings of 25th International Technical Meeting of the Satellite Division of the Institute of Navigation (ION GNSS 2012), Nashville, TN 2012, pp. 743-763

10. Sabatini, R., Moore, T., Hill, C., Ramasamy, S.: Assessing avionics-based GNSS integrity augmentation performance in UAS mission-and safety-critical tasks. In: Unmanned Aircraft Systems (ICUAS), 2015 International Conference on 2015, pp. 650-659. IEEE

11. Liso Nicolas, M., Jacob, M., Smyrnaios, M., Schon, S., Kurner, T.: Basic concepts for the modeling and correction of GNSS multipath effects using ray tracing and software receivers. In: Antennas and Propagation in Wireless Communications (APWC), 2011 IEEE-APS Topical Conference on 2011, pp. 890-893. IEEE

12. Iskander, M.F., Yun, Z.: Propagation prediction models for wireless communication systems. Microwave Theory and Techniques, IEEE Transactions on 50(3), 662-673 (2002).

13. Pirjanian, P.: An overview of system architecture for action selection in mobile robotics. Laboratory of Image Analysis, Aalborg University, Aalborg, Denmark (1997).

14. Gat, E.: On three-layer architectures. Artificial intelligence and mobile robots 195, 210 (1998).

15. Rezaei, S., Sengupta, R.: Kalman filter-based integration of DGPS and vehicle sensors for localization. Control Systems Technology, IEEE Transactions on 15(6), 1080-1088 (2007).

16. Sabatini, R., Rodriguez, L., Kaharkar, A., Bartel, C., Shaid, T.: Carrier-phase GNSS attitude determination and control system for unmanned aerial vehicle applications. ARPN Journal of Systems and Software 2(11), 297-322 (2012).

17. Sabatini, R., Bartel, C., Kaharkar, A., Shaid, T., Rodriguez, L., Zammit-Mangion, D., Jia, H.: Low-Cost Navigation and Guidance Systems for Unmanned Aerial Vehicles-Part 1: VisionBased and Integrated Sensors. Annual of Navigation 19(2), 71-98 (2012).

18. Kaplan, E., Hegarty, C.: Understanding GPS: principles and applications. Artech house, (2005)

19. Parkinson, B.W., Spilker, J.J.: Global positioning system: theory and practice. Volumes I and II., Washington DC, American Institute of Aeronautics and Astronautics Inc (1996).

20. Sabatini, R., Rodríguez, L., Kaharkar, A., Bartel, C., Shaid, T., Zammit-Mangion, D.: Low-Cost Navigation and Guidance Systems for Unmanned Aerial Vehicles-Part 2: Attitude 
This is the author pre-publication version. This paper does not include the changes arising from the revision, formatting and publishing processes. The final version that should be used for referencing is:

S. Bijjahalli, S. Ramasamy and R. Sabatini, "A Novel Vehicle-Based GNSS Integrity Augmentation System for Autonomous Airport Surface Operations", Journal of Intelligent \& Robotic Systems, pp. 1-25, 2017. DOI: 10.1007/s10846-017-0479-8

Determination and Control. Annual of Navigation 20(1), 97-126 (2013).

21. Purcell Jr, G., Srinivasan, J., Young, L., DiNardo, S., Hushbeck Jr, E., Meehan, T., Munson, T., Yunck, T.: Measurement of Aircraft Position, Velocity, and Attitude Using Rogue GPS Receivers. In: Proceedings of the 5th International Geodetic Symposium on Satellite Positioning, Las Cruces, NM 1989

22. GRAAS, F., Braasch, M.: GPS interferometric attitude and heading determination: Initial flight test results. Navigation 38(4), 297-316 (1991).

23. Cohen, Clark Emerson. "Attitude determination using GPS." PhD thesis Department of Aeronautics and Astronautics, Stanford University (1992).

24. Hofmann-Wellenhof, B., Lichtenegger, H., Wasle, E.: GNSS-global navigation satellite systems: GPS, GLONASS, Galileo, and more. Springer Science \& Business Media, (2007)

25. Sabatini, R., Palmerini, G.: Differential Global Positioning System (DGPS) for Flight Testing. In: Organisation, R.a.T. (ed.) AGARDograph 160,Flight Test Instrumentation Series, Research and Technology Organisation, (2008)

26. Feng, S., Ochieng, W., Moore, T., Hill, C., Hide, C.: Carrier phase-based integrity monitoring for high-accuracy positioning. GPS solutions 13(1), 13-22 (2009).

27. Pervan, B.S., Lawrence, D.G., Parkinson, B.W.: Autonomous fault detection and removal using GPS carrier phase. Aerospace and Electronic Systems, IEEE Transactions on 34(3), 897-906 (1998).

28. Michalson, W., Hua, H.: GPS carrier-phase RAIM. In: Proceedings of the 8th International Technical Meeting of the Satellite Division of The Institute of Navigation (ION GPS 1995) 1995, pp. 1975-1984

29. Chang, X.-w., Paige, C., Perepetchai, V.: Integrity methods using carrier phase. (2001).

30. Beutler, G., Davidson, D., Langley, R., Santerre, R., Vanicek, P., Wells, D.: Some theoretical and practical aspects of geodetic positioning with the Global Positioning System using carrier phase difference observations, Dept. In. of Surveying Engineering Technical Report, (1984)
31. Dai, Z.: On GPS based attitude determination. Universitätsbibliothek der Universität Siegen (2013)

32. Altmayer, C.: Enhancing the integrity of integrated GPS/INS systems by cycle slip detection and correction. In: Intelligent Vehicles Symposium, 2000. IV 2000. Proceedings of the IEEE 2000, pp. 174-179. IEEE

33. El-Sheimy, N.: An expert knowledge GPS/INS system for mobile mapping and GIS applications. 2000: Navigating into the New Millennium, 816-824 (2000).

34. Sabatini, R., Moore, T., Hill, C.: A New Avionics-Based GNSS Integrity Augmentation System: Part 2-Integrity Flags. Journal of Navigation 66(04), 501-522 (2013).

35. Ramasamy, S., Sabatini, R., Gardi, A.: Towards a unified approach to cooperative and noncooperative RPAS detect-and-avoid. In: Fourth Australasian Unmanned Systems Conference 2014

36. Gardi, A., Sabatini, R., Ramasamy, S., de Ridder, K.: 4-Dimensional Trajectory negotiation and validation system for the next generation air traffic management. In: proceedings of AIAA Guidance, Navigation, and Control Conference (GNC 2013), Boston, MA, USA 2013

37. Lau, L., Cross, P.: Development and testing of a new ray-tracing approach to GNSS carrier-phase multipath modelling. Journal of Geodesy 81(11), 713-732 (2007).

38. Hata, M.: Empirical formula for propagation loss in land mobile radio services. IEEE transactions on Vehicular Technology 29(3), 317-325 (1980).

39. Okumura, Y., Ohmori, E., Kawano, T., Fukuda, K.: Field strength and its variability in VHF and UHF land-mobile radio service. Rev. Elec. Commun. Lab 16(9), 825-873 (1968).

40. Moore T, Hill C, Hide C, Cross P, Lau L, Walsh $\mathrm{D}$, Cooper J, Ioannides R, Ochieng W, Feng S (2005) Development of a test bed facility for high accuracy positioning in difficult environments. In: Proceedings of ION GNSS 2005, The Institute of Navigation, Long Beach, 13-16 September.

41. Kim, D.-Y., Jang, J.-G., Kee, C.-D.: Integer Ambiguity Search Technique Using SeparatedGaussian Variables. International 
This is the author pre-publication version. This paper does not include the changes arising from the revision, formatting and publishing processes. The final version that should be used for referencing is:

S. Bijjahalli, S. Ramasamy and R. Sabatini, "A Novel Vehicle-Based GNSS Integrity Augmentation System for Autonomous Airport Surface Operations", Journal of Intelligent \& Robotic Systems, pp. 1-25, 2017. DOI: 10.1007/s10846-017-0479-8

Journal of Aeronautical and Space Sciences 5(2), 1-8 (2004).

42. Möller, T., Trumbore, B.: Fast, MinimumStorage Ray-Triangle Intersection; 1997. Journal of Graphic Tools (2014).

43. Lvovsky, Alexander I. "Fresnel equations Encyclopedia of Optical Engineering." Taylor and Francis: New York, Published online 27 (2013), pp. 1-6.

44. Kraus, J.D., Fleisch, D.A.: Eletromagnetics: With Applications. WCB/McGraw-Hill, (1999).

45. Ward, P.W.: GPS receiver RF interference monitoring, mitigation, and analysis techniques. Navigation 41(4), 367-392 (1994).

46. Braasch, M.S., Van Dierendonck, A.: GPS receiver architectures and measurements. Proceedings of the IEEE 87(1), 48-64 (1999).

47. Dodson, A.H.: Propagation effects on GPS measurements. In. Institute of Engineering,Surveying and Space Geodesy University of Nottingham, Nottingham,United Kingdom, (2002)

48. Collins, J.P.: Assessment and development of a tropospheric delay model for aircraft users of the global positioning system. University of New Brunswick Fredericton, New Brunswick (1999)

49. Black, H., Eisner, A.: Correcting satellite Doppler data for tropospheric effects. Journal of Geophysical Research: Atmospheres 89(D2), 2616-2626 (1984).

50. Friis, H.T.: The Free Space Transmission equation. Proc. IRE 34, 254 (1946).

51. Mubarak, O.M., Dempster, A.G.: Statistical analysis of early late phase for multipath detection. In: IGNSS Symposium 2009.

52. RTCA Special Committee 159, DO 229D: "Minimum Operational Performance Standards for Global Positioning System/Wide Area Augmentation System Airborne Equipment", Radio Technical Commission for Aeronautics (RTCA) (2006).

53. RTCA DO245A: " Minimum aviation system performance standards for the local area augmentation system (LAAS)", Technical Standard, DO245A, Radio Technical Commission for Aeronautics (RTCA) (2004).

54. Sabatini, R., Moore, T., Hill, C., Ramasamy, S.: Investigation of GNSS Integrity Augmentation Synergies with Unmanned Aircraft Sense-and-
Avoid Systems. In. SAE Technical Paper, (2015).

55. Will, A.B., $Z^{\circ}$ AK, S.H.: Modelling and control of an automated vehicle. Vehicle System Dynamics 27(3), 131-155 (1997).

56. Jazar, R.N.: Vehicle dynamics: theory and application. Springer Science \& Business Media, (2013).

57. Ulsoy, A.G., Peng, H., Çakmakci, M.: Automotive control systems. Cambridge University Press, (2012).

58. Kelso, T.: CelesTrak. Public Domain Satellite Tracking Data, URL: http://celestrak. com/[cited 13 March 2012] (2006).

\section{Author Biographies}

Mr. Suraj Bijjahalli obtained his Master in Automobile Engineering from RMIT University (Australia). Prior to pursuing his postgraduate degree, he was working in the automotive industry in India (TATA Motors Limited) where he gained hands-on experience in Field Service Management for cars. He is currently pursuing his doctoral degree at RMIT University in the area of GNSS integrity augmentation systems targeting Unmanned Aircraft System (UAS) operations in urban environments. His areas of interest are GNSS performance augmentation, aviation safety, lowcost navigation and guidance sensors, multi-sensor data fusion and UAS traffic management.

Mr. Subramanian Ramasamy obtained his postgraduate degree in Aerospace Engineering (specialisation in Avionics) from Madras Institute of Technology (India). Prior to pursuing his $\mathrm{PhD}$, he was working at THALES, a French multinational aerospace and defence company, gaining hands-on experience in Flight Management Systems and Human Machine Interfaces. Subramanian started his doctoral studies at Cranfield University (UK), where he was part of the Systems for Green Operations Integrated Technology Demonstrator (SGO-ITD) of the Clean Sky Joint Technology Initiative (JTI) for Aeronautics and Air Transport research group and is currently continuing his work at RMIT University (Australia). His areas of interest are aeronautical Communication, Navigation and Surveillance (CNS) systems, 
This is the author pre-publication version. This paper does not include the changes arising from the revision, formatting and publishing processes. The final version that should be used for referencing is:

S. Bijjahalli, S. Ramasamy and R. Sabatini, "A Novel Vehicle-Based GNSS Integrity Augmentation System for Autonomous Airport Surface Operations", Journal of Intelligent \& Robotic Systems, pp. 1-25, 2017. DOI: 10.1007/s10846-017-0479-8

Unmanned Aircraft Systems (UAS) Traffic Management (UTM) and Cognitive Human Machine Interface and Interactions for aerospace applications.

Roberto Sabatini is a Professor of Aerospace Engineering and Aviation at RMIT University (Australia) with more than 25 years of experience in the aerospace/defence industry and in academia. He is an expert in Air Traffic Management (ATM), avionics and Unmanned Aircraft Systems (UAS), with specific hands-on competence in aeronautical Communication, Navigation and Surveillance (CNS), flight Guidance, Control and Dynamics (GCD), aviation human factors engineering (cognitive HMI, human-machine teaming and trusted autonomy), and multi-sensor data fusion for civil and military applications. Currently, Prof. Sabatini is the Head of the Intelligent \& CyberPhysical Transport Systems Research Group and the Aviation Program Leader at RMIT University. Prof. Sabatini's current research focus is on integrated CNS/ATM and Avionics (CNS+A) systems for improved aviation safety, efficiency and environmental sustainability. Prof. Sabatini received several scientific and academic awards including the SARES Science Award (2016), the SAE Arch T. Colwell Merit Award (2013), and the NATO Research \& Technology Organisation Scientific Achievement Award (2008). Prof. Sabatini is a Fellow of the Royal Institute of Navigation, a Senior Member of IEEE, a Senior Member of AIAA, and a Life Member of AFCEA. 